\title{
Bioaccumulation of non-essential trace metals in tissues and organs of Clarias gariepinus (sharptooth catfish) from the Vaal River system - strontium, aluminium, lead and nickel
}

\author{
D Crafford ${ }^{1}$ and A Avenant-Oldewage $\mathrm{e}^{2 *}$ \\ ${ }^{1}$ Department of Zoology, University of Johannesburg, PO Box 524, Auckland Park, Johannesburg, 2006, South Africa. \\ Current address: Clinvet International Pty. Ltd., PO Box 11186, Universitas, 9321, South Africa \\ ${ }^{2}$ Department of Zoology, University of Johannesburg, PO Box 524, Auckland Park, Johannesburg, 2006, South Africa
}

\begin{abstract}
This is the first paper reporting on results obtained in a metal bioaccumulation study in the Vaal River system. It discusses concentrations of four non-essential elements (strontium, aluminium, lead and nickel) in water, sediment and various fish tissues. A second paper will report on concentration levels of the remaining five essential trace metals (chrome, copper, iron, manganese and zinc) studied. While heavy metals did accumulate in C. gariepinus tissues, no clear trends emerged with regard to differences between localities (Vaal Dam and Vaal River Barrage) or surveys. The highest non-essential element metal concentrations were generally recorded in gill (filaments and arches), followed by muscle, liver and lastly skin. This general trend appears to be in agreement with trends observed by other workers and reported in the literature. Variability in tissue metal concentrations in C. gariepinus within locality and seasons observed in this study is also reflected in results from available literature. This accentuates the importance of factors that influence the concentrations and bioavailability of trace metals.
\end{abstract}

Keywords: Heavy metal pollution, bioaccumulation, Clarias gariepinus, Vaal River Barrage, Vaal Dam, strontium, aluminium, lead, nickel

\section{Introduction}

\section{Synopsis}

With South Africa's unallocated water supplies dwindling (Blignaut and van Heerden, 2009), pollution levels (including heavy metal pollution) will influence future water conservation and management policy-making decisions. Furthermore, metal pollution may globally have a severe negative impact on human health (Castro-González and Méndez-Armenta, 2008), with South Africa being no exception (Strydom et al., 2006). In fish, lesions caused by low concentrations of trace metals could lead to functional alterations and interference with fundamental processes such as osmoregulation, gas exchange and metabolism (Pandey et al., 2008), and subsequently also affect community structure (Bervoets et al., 2005). Heavy metals can be defined as 'electropositive elements having a density of greater than five' (Roebuck, 1992). This definition invariably lumps together 40 metals of different properties and degrees of acute and chronic toxicity (Roebuck, 1992). With regards to the periodic table, the transition elements are generally termed 'heavy metals' or simply 'metals' (Sorensen, 1991). Possible sources include wastewater arising from informal settlements (Jackson et al., 2007), leachates from domestic and industrial landfill sites (Moodley et al., 2007), mining activities, disposal of metal-containing industrial effluents (Lloyd,

\footnotetext{
* To whom all correspondence should be addressed.

正 +2711 559 2449; fax: +2711 559 2286; e-mail: aoldewage@uj.ac.za

Received 28 February 2010; accepted in revised form 3 September 2010.
}

1992; Purves, 1985; Phuong et al., 1998), municipal wastewater, dry docking companies and petrol filling stations (Shriadah, 1998). Presence of metals in natural waters may either result in positive effects when the metals present are essential for life in low concentrations, or negative (i.e. toxicologically undesirable) effects (Galvin, 1996). Toxicity of metals is dependent on the availability of the metal in the ionised form (Sorensen, 1991). This in turn is influenced by factors such as hardness, $\mathrm{pH}$, dissolved oxygen, temperature, salinity, interactions with heavy metal salts and other particles such as suspended solids (Hartung, 1973; Mance, 1987; Wang, 1987; Burton et al., 1972). Synergistic combined effects or additive joint actions of metals have been documented (Enserink et al., 1991; Palaniappan and Karthikeyan, 2009). Antagonistic interactions also occur (e.g. Kwong and Niyogi, 2009). Sorption of several metals to hydrous ferric oxide (Johnson, 1986; Hem, 1977; Swallow et al., 1980) and manganese oxide (Loganathan and Burau, 1973) has been studied. Another factor affecting toxicity of metals is alkalinity (Köck et al., 1995). It is, however, not only water quality variables and chemical elements that are important determinants of metal availability and toxicity. A large number of biological variables also play a significant role with regard to metal accumulation. These include interspecies variations (Giesy and Wiener, 1977; Lowe et al., 1985), orientation to the sediment and behaviour (Kligour, 1991; Kidwell et al., 1995), as well as life stages present (Mance, 1987). Synthetic chelating agents (e.g. ethylene diamine tetraacetic acid) can also reduce metal toxicity (Muramoto, 1980; James et al., 1998). Effects of metal toxicity are varied, ranging from gross pathologies like scale deformation (Yoshitomi et al., 1998) and liver degeneration (Woodward et al., 1994), to the disruption of metabolic 
processes (Barnhoorn et al., 1999) and behaviour (e.g. spawning) (Benoit, 1975).

Metal accumulation is affected by some of the same parameters that affect toxicity (water chemistry and particulate matter). As such it is potentially one of the most valuable tools for identifying and quantifying the impact of metals in aquatic environments (Borgmann and Norwood, 1994; Birungi et al., 2007; Shinn et al., 2009; Allert et al., 2009; Otero-Muras et al., 2010). This impact has been widely investigated in studies examining accumulation in sediments (Rosales-Hoz and Carranza-Edwards, 1998), crustaceans (Zou, 1997; Steenkamp et al., 1994), aquatic insects (Hall et al., 1998), amphipods (Borgmann and Norwood, 1994), mammals associated with water (Gutleb et al., 1998), aquatic macrophytes (van der Merwe et al., 1990), algae (Carr et al., 1998), plankton (Ghatak et al., 1987) and several species of fish (Maage, 1990; Sultana and Rao, 1998; Ward and Neumann, 1999; Annune et al., 1994; Lange et al., 1993; Neuman et al., 1991; Prasath and Khan, 2008). The fish tissues examined to a large extent depend on the objective of the research. If the potential effect of consumption of fish on human health is to be determined the tissue analysed is usually muscle, with the skin either included or removed. When the objective of the research is to determine effects of a contaminant on individual fish health, organs such as kidney, gill or liver are analysed. Whole body analysis will be performed if the objectives include trophic interactions, bioconcentration and bioaccumulation (Goldstein et al., 1995). In biomagnification studies both tissue samples and whole body analysis are often employed, as was demonstrated by Retief et al. (2009). They performed selective host tissue and whole body parasite analysis to examine metal accumulation in the cestode Bothriocephalus acheilognathi. The parasite had higher metal concentrations than host tissue, indicating the potential for biomagnification.

Absorption of heavy metals can occur via 2 pathways, as discussed by Bryan (1976) and demonstrated in a comparative study by Alquezar et al. (2008). The first is absorption from solution. Ion transfer through the gills serves as a good example. Metals may, however, also diffuse passively through skin and gills as a soluble complex down gradients created by adsorption at the surface. The second pathway is absorption from food or particles. After reviewing available literature, Bryan (1976) came to the conclusion that, in the majority of cases, food is a much more important source of metals than the water. After these trace elements are absorbed, it is transferred from the gills and intestine to the blood and distributed to other parts of the body (Hogstrand and Haux, 1991).

Essential trace metals (like zinc and copper) are better regulated than nonessential trace metals (like lead and cadmium), since detoxification of nonessential elements may involve sequestering, compared to elimination of essential elements (Giesy and Wiener, 1977). As a result zinc is believed not to accumulate in food chains (van den Heever and Frey, 1994), being lost primarily via the gills (Bryan, 1976). Some metals are also excreted into bile (Klaasen, 1976). Excretion via both the gills and biliary route seem to be quantitatively more important than the urinary route (Klaasen, 1976; Bryan, 1976). Metallothionein, a low molecular weight protein, also plays a role in the binding and detoxification of heavy metals (Hodson, 1988; Hogstrand and Haux, 1991). It has been found that nonessential elements, on the other hand, tend to accumulate in fish year to year in a stepwise manner, since fish are not able to eliminate the metals completely (Köck et al., 1996). In fact, Sorensen (1991) states:
'Loading of aquatic habitats with nondegradable, nonnutritive, cumulative pollutants such as $\mathrm{As}, \mathrm{Pb}, \mathrm{Cd}$ and $\mathrm{Hg}$ can result in undeniably complex alterations of numerous teleost trophic levels'. All metals, however, are elements and hence cannot disappear from a system, but can only be transferred from one place to another (Lloyd, 1992). It is the build-up of these metals over many decades in sediment 'sinks' that is of major concern today (Lloyd, 1992). Sediment can affect water quality in many ways. Sorption of pollutants on sediments alters their positional- and bioavailability, and under certain circumstances pollutants can be remobilised (Coetzee, 1993; Grobler et al., 1987). A number of mechanisms regarding metal binding to, and release from, sediments have been identified. These include acid-volatile sulphides in anaerobic sediments, particulate organic carbons in aerobic sediments, iron and manganese oxyhydroxides in aerobic sediments (all metal-binding phases), complexation by ligands and oxidation caused by physical, biological and human activities (Chapman et al., 1998), such as re-suspension of sediment during flood conditions (Literathy and Laszlo, 1977).

Water bodies in the Vaal River system are generally shallow and seldom develop anaerobic hypolimnia (Grobler et al., 1987). As a result re-suspension (i.e. oxidation caused by physical and biological activities) rather than chemical release could be the dominant mechanism for returning pollutants to the water column (Grobler et al., 1987). Gouws and Coetzee (1997) found the extractable metal content ( $\mathrm{Ni}, \mathrm{Mn}, \mathrm{Co}, \mathrm{Cr}$, $\mathrm{Zn}, \mathrm{Fe}, \mathrm{Ca}, \mathrm{Sn}, \mathrm{Cd}, \mathrm{Pb}, \mathrm{Al}$ and $\mathrm{Cu}$ ) of Vaal Dam sediment to be low. Furthermore major proportions of most metals seemed to be associated with the inert phase and could therefore be classified as being of geochemical origin (Gouws and Coetzee, 1997).

\section{Scope of study}

In the current study the concentrations of 9 metals (strontium, aluminium, chromium, copper, zinc, manganese, iron, lead and nickel) were determined in 5 tissue types, namely, skin, muscle, gill arch, gill filament and liver of the sharptooth catfish (Clarias gariepinus). In doing so the possible effect of consumption on human health, as well as effects on fish health, could be discussed. Ultimately the range of tissues used also provided a good indication of the degree of bioaccumulation by the sampled fish populations. Accumulation of trace metals is influenced by feeding behaviour (e.g. Ali and Fishar, 2005). In this respect catfish are particularly suited to bioaccumulation studies. Bottom feeders are readily exposed to metals that accumulate in sediment, while predators accumulate metals from surrounding water or from feeding on other fish (Kidwell et al., 1995). Catfish are bottom-dwelling omnivores, but also actively feed on a wide variety of prey including small fish (Skelton, 2001).

The data gathered during the study was compared between a polluted (Vaal River Barrage affected by industrial, mining and domestic effluent) and less polluted (Vaal Dam located in the upper catchment) locality. Trace metal concentrations in sediment and water samples (Institute for Water Quality Studies, Department of Water Affairs and Rand Water Board database) were also recorded during each survey.

Of the 9 metals for which analysis was performed, chromium $(\mathrm{Cr})$, copper $(\mathrm{Cu})$, iron $(\mathrm{Fe})$, manganese $(\mathrm{Mn})$, and zinc (Zn) are regarded as essential trace metals (Galvin, 1996). These metals play a vital role in many physiological processes, but have a toxic effect when present in high enough 
concentrations. Aluminium (Al), lead (Pb), nickel (Ni) and strontium (Sr), on the other hand, are considered to be nonessential elements. This, the first paper reporting on results obtained in the study, shall deal only with the latter.

\section{Strontium}

The chemistry of strontium ( $\mathrm{Sr}$ ) is similar to that of calcium (Hem, 1989). As such it can be of use in the field of freshwater fisheries management. Schroder et al. (1995) used rings of deposited strontium in otoliths as tags in salmon fry. Strontium was also found to accumulate in the vertebrae and opercula. Radtke (1989) also proposed the use of strontium-calcium concentration ratios in otoliths as environmental indicators. Strontium can affect fish adversely at elevated levels, but, prior to a short review of available literature, Seymore et al. (1995) stated that limited research has been conducted in this field.

\section{Aluminium}

Aluminium (Al) is one of the more common elements in the earth's crust ( $8.1 \%$ by mass) (Galvin, 1996). Dissolved aluminium concentrations are inversely correlated with $\mathrm{pH}$ (i.e. high dissolved aluminium concentrations in waters with high acid loads) (Dickson, 1983; Baker and Schofield, 1982). Dissolved aluminium is also more toxic in acidic environments (Buergel and Soltero, 1983; Norrgren et al., 1991). Metal accumulation is affected by some of the same parameters that affects toxicity (Borgmann and Norwood, 1994), and as such the two should be considered together.

\section{Lead}

Lead $(\mathrm{Pb})$ is widely used as an additive in (among others) some fuels, production of batteries, paints, anti-radiation armour and pesticide formulations (Galvin, 1996). Sorensen (1991) describes $\mathrm{Pb}$ as a 'bone-seeking' element. Lead poisoning in fish result in haematological (e.g. higher blood glucose levels), neuronal, muscular and other effects like black tails, lordoscoliosis, pigment alterations and coagulation of surface mucus (Sorensen, 1991; Haux et al., 1986). It can also result in a delay in larval development (Benijts-Claus and Benijts, 1975). Toxicity of $\mathrm{Pb}$ is reduced in hard water (i.e. high concentration of $\left.\mathrm{CaCO}_{3}\right)($ Galvin, 1996) as Ca has the ability to antagonise some of the toxic effects of $\mathrm{Pb}$ (Roebuck, 1992). Furthermore $\mathrm{Pb}$ accumulation exhibits a negative correlation with $\mathrm{pH}$ (Merlini and Pozzi, 1977).

\section{Nickel}

Nickel (Ni) is used in the melting process of metallic alloys, as catalyst and in pesticide formulations (Galvin, 1996). Other sources include alkaline storage batteries and the production and utilisation of coal (Birge and Black, 1980). The metal is generally considered not to be very toxic to man (Galvin, 1996) since it is regarded as an essential element (Moore and Ramamoorthy, 1984). Certain salts can however have potentially carcinogenic and mutagenic effects through interactions between the metal and cellular DNA (Galvin, 1996). In humans high ingestion of Ni can also cause renal problems and skin allergies by contact (Galvin, 1996). As is the case with other essential elements like $\mathrm{Zn}$, $\mathrm{Ni}$ is toxic to fish when present in high enough concentrations (Pickering, 1974).

\section{Aim}

The objectives of this study were to determine to what extent:

- Heavy metal accumulation occurs in Clarias gariepinus tissues, and in which tissues (i.e. ranking concentrations in skin, muscle, gill arch, gill filament and liver)

- Metal concentrations differ between localities (Vaal Dam and Vaal River Barrage)

- Metal concentrations differ between surveys and seasons

\section{Materials and methods}

\section{Study sites}

Surveys were conducted bimonthly at 2 localities: the former RAU Island (now UJ Island) in the Vaal Dam (S 26 52.249', E $\left.28^{\circ} 10.249^{\prime}\right)$ and the Vaal River Barrage near the Barrage wall (S $26^{\circ} 45.786^{\prime}$, E $27^{\circ} 41.280^{\prime}$ ). Water quality in the Vaal River Barrage is poorer than that of the Vaal Dam, due to increased levels of urban, industrial, mining and agricultural effluent and runoff. Surveys were conducted during the following months: November 1998, January 1999, March 1999, June 1999, August 1999, October 1999 and February 2000.

\section{Water and sediment analyses}

Integrated water samples from 0 to $5 \mathrm{~m}$ were obtained with the aid of a modified hosepipe. Sediment samples were obtained with the aid of a sediment corer. The Department of Water Affairs' Institute for Water Quality Studies (Roodeplaat Dam, Pretoria) performed all water and sediment trace metal analyses. Rand Water Board also made data available on trace metal analyses resulting from their monitoring activities. Data from the following Rand Water Board sampling points were used: C-VD1I (Rand Water Board Inlet at the Vaal Dam wall) and C-VRB0T (upstream at the Vaal River Barrage).

\section{Tissue and organ collection}

During each survey 20 Clarias gariepinus (Burchell, 1822) were collected at both localities with the aid of gill nets (stretched mesh sizes 90, 110 and $130 \mathrm{~mm}$.). Fish were transported back to land in a live well and transferred to a holding tank through which dam or river water was continuously circulated. Fish were weighed, measured, placed on a polypropylene dissection board and killed by severing the spinal cord behind the head. The following tissues or organs were removed: liver, skin, muscles and gills (filaments attached to arch). Enough tissue was collected to fill the $25 \mathrm{~m} \ell$ glass bottle in which it was stored. Samples were kept on ice before being transferred to a deep freeze at the end of each day. The frozen samples were transported to the laboratory.

\section{Laboratory analysis}

In the laboratory samples were allowed to thaw at room temperature. Approximately $5 \mathrm{~g}$ of wet tissue (liver, skin and muscle) were weighed in an Erlenmeyer flask with the aid of a Mettler PK 4800 scale. This corresponded to approximately $1 \mathrm{~g}$ dry weight. All glassware was soaked in $1 \mathrm{M}$ hydrochloric acid (HCL) for $24 \mathrm{~h}$ and rinsed in doubly-distilled water prior to use (Giesy and Wiener, 1977). The samples were subsequently placed in a Haraeus Hanau KB 500 oven at $60^{\circ} \mathrm{C}$ for $48 \mathrm{~h}$ or until completely dehydrated. In previous studies (Marx, 1996) 
only the gill filaments were used for metal analysis after being removed from the arches using small scissors. Seymore et al. (1995) suggested that bony tissue should be included in metal accumulation studies. As a result both gill filaments and gill arches were used in the current study. It was found that not all filament tissue could be successfully removed from the arches using dissection equipment. Whole gill arches with filaments still attached were placed in the Haraeus Hanau KB 500 oven until completely dehydrated. Once dehydrated the filaments could effortlessly be removed from the arches. The dehydrated skin, liver and muscle tissue were weighed again and the weight in grams noted. Dehydrated gill filament $(1 \mathrm{~g})$ and gill arch tissue ( $1 \mathrm{~g}$ ) were weighed out and the exact weight was recorded. A 2:1 mixture of 55\% nitric acid $\left(\mathrm{NHO}_{3}\right)$ and $70 \%$ perchloric acid $\left(\mathrm{HCLO}_{4}\right)$ was then added to the $1 \mathrm{~g}$ of dried tissue in the Erlenmeyer flask (Van Loon, 1980; Houba et al., 1983). Marx (1996) used a mixture of $10 \mathrm{~m} \ell \mathrm{NHO}_{3}$ and $5 \mathrm{~m} \ell$ $\mathrm{HCLO}_{4}$. In the current study a mixture of $14 \mathrm{~m} \ell \mathrm{NHO}_{3}$ and $7 \mathrm{~m} \ell \mathrm{HCLO}_{4}$ was used. This allowed the complete digestion of most tissue samples without having to add more of the acid mixture due to evaporation. The results are still comparable, however, since the formula used allows for the subtraction of acid metal concentrations. After adding the acid mixture the samples were placed on a hot plate at approximately $200^{\circ} \mathrm{C}$ and allowed to digest until transparent (Van Loon, 1980). After the digested samples were removed from the hot plate and allowed to cool to room temperature, they were diluted to $50 \mathrm{~m} \ell$ with distilled water. These samples were subsequently filtered individually with the aid of a $6 \mu \mathrm{m}$ Millipore acid-resistant membrane filter attached to a vacuum pump. The filtered samples were poured into acid-washed glass bottles rinsed in distilled water. Following each filtration the filter system and membrane pump were also rinsed in distilled water. The total metal concentrations of $\mathrm{Sr}, \mathrm{Al}, \mathrm{Pb}$ and $\mathrm{Ni}$ were determined with the aid of a Varian Atomic Absorption Spectrophotometer (SPECTRA AA-10). For each metal, Holpro stock solutions were used to prepare 4 different concentrations of analytical standards. Calibration of the spectrophotometer, for each metal individually, was subsequently conducted with the aid of these standards. Individual concentrations of the digested tissue samples were then read against particular absorbencies defined for each metal. An Air-Acetylene flame was used to read strontium and aluminium, while an Air-Nitrous flame was used for all other metals. Both strontium and aluminium was only analysed after adding $0.5 \mathrm{~m} \ell$ of a $2.682 \mathrm{M}$ potassium chloride ( $200 \mathrm{~g} \mathrm{KCl}$ per $1000 \mathrm{~m} \ell$ distilled water) to the $50 \mathrm{~m} \ell$ sample. Potassium chloride is added to keep these metals from ionising (Varian, 1989). A 2:1 (14 m $: 7 \mathrm{m \ell})$ solution of nitric and phosphoric acid was also made up and its absorbency read using the spectrophotometer. The metal concentrations thus determined could be subtracted from the concentrations determined for the digested $50 \mathrm{~m} \ell$ samples, using the following formula:

$$
\begin{gathered}
\text { Metal } \\
\begin{array}{c}
\text { concentration } \\
(\mu \mathrm{g} / \mathrm{g})
\end{array}
\end{gathered}=\frac{\text { AAS reading }(\mu \mathrm{g} / \mathrm{m} \ell)}{\text { Sample mass }(\mathrm{g}) \text { dry wt. }} \times \begin{gathered}
\text { Sample volume } \\
(50 \mathrm{~m} \ell)
\end{gathered}-\begin{gathered}
\text { Acid metal } \\
\text { concentration }
\end{gathered}
$$

Statistical significance of metal concentrations between localities and surveys respectively was investigated and tabulated as T-test p-values.

\section{Results}

\section{Strontium}

Water and sediment

\begin{tabular}{|c|c|c|c|}
\hline \multicolumn{4}{|c|}{$\begin{array}{c}\text { Table } 1 \\
\text { Strontium concentrations in water and sediment of the Vaal } \\
\text { Dam and Vaal River Barrage, obtained during } 7 \text { surveys } \\
\text { conducted between } 1998 \text { and } 2000\end{array}$} \\
\hline \multirow[t]{2}{*}{ Month of survey } & \multirow{2}{*}{$\begin{array}{l}\text { Water or sediment } \\
\text { sample }\end{array}$} & \multicolumn{2}{|c|}{ Locality } \\
\hline & & Vaal Dam & $\begin{array}{c}\text { Vaal River } \\
\text { Barrage }\end{array}$ \\
\hline \multirow[t]{2}{*}{ November (1998) } & Water $(\mathrm{mg} / \ell)$ & N/A & N/A \\
\hline & Sediment (mg/g) & 0.277 & N/A \\
\hline \multirow[t]{2}{*}{ January (1999) } & Water $(\mathrm{mg} / \ell)$ & N/A & N/A \\
\hline & Sediment (mg/g) & 0.184 & 0.161 \\
\hline \multirow[t]{2}{*}{ March (1999) } & Water $(\mathrm{mg} / \ell)$ & N/A & N/A \\
\hline & Sediment (mg/g) & 0.116 & 0.165 \\
\hline \multirow[t]{2}{*}{ June (1999) } & Water $(\mathrm{mg} / \ell)$ & N/A & 0.168 \\
\hline & Sediment (mg/g) & 0.207 & 0.165 \\
\hline \multirow[t]{2}{*}{ August (1999) } & Water $(\mathrm{mg} / \ell)$ & 0.125 & 0.181 \\
\hline & Sediment (mg/g) & 0.111 & 0.371 \\
\hline \multirow[t]{2}{*}{ October (1999) } & Water $(\mathrm{mg} / \ell)$ & 0.146 & 0.204 \\
\hline & Sediment (mg/g) & 0.054 & 0.296 \\
\hline \multirow[t]{2}{*}{ February (2000) } & Water $(\mathrm{mg} / \ell)$ & 0.106 & 0.138 \\
\hline & Sediment (mg/g) & 0.231 & 0.088 \\
\hline
\end{tabular}


Differences in Sr concentrations recorded from sediment and water between surveys were not reflected in concentrations recorded from fish tissues. In the Vaal Dam $\mathrm{Sr}$ was accumulated in the different tissues (Table 2), in order of decreasing concentration, as follows: gill arch $>$ gill filament $>$ skin $>$ liver
$>$ muscle. In the Vaal River Barrage, the decreasing order of $\mathrm{Sr}$ concentrations in tissues was: gill arch $>$ gill filament $>$ muscle $\geq$ liver $\geq$ skin. No clear trends regarding seasonal Sr concentrations could be identified (Table 3 ).

Table 2

Strontium concentrations $(\mu \mathrm{g} / \mathrm{g})$ in 5 different tissue types of Clarias gariepinus from the Vaal Dam and Vaal River Barrage (shaded blocks). T-test $p$-values indicate whether concentrations recorded in each tissue type differed significantly between localities.

\begin{tabular}{|c|c|c|c|c|c|c|c|c|}
\hline \multirow[t]{2}{*}{ Month } & \multirow[t]{2}{*}{ Locality } & \multirow{2}{*}{$\begin{array}{l}\text { Statistical } \\
\text { variable }\end{array}$} & \multirow[b]{2}{*}{ Skin } & \multirow[b]{2}{*}{ Liver } & \multicolumn{3}{|c|}{ Tissue type } & \multirow[b]{2}{*}{\begin{tabular}{|l} 
Whole \\
gill
\end{tabular}} \\
\hline & & & & & Muscle & Gill arch & $\begin{array}{l}\text { Gill } \\
\text { filament }\end{array}$ & \\
\hline November (1998) & $\begin{array}{l}\text { Vaal Dam } \\
(\mathrm{n}=20)\end{array}$ & $\begin{array}{l}\text { Mean } \\
\text { Std. deviation }\end{array}$ & $\begin{array}{l}2.23 \\
1.76\end{array}$ & $\begin{array}{l}2.94 \\
2.32\end{array}$ & $\begin{array}{l}1.11 \\
1.22\end{array}$ & - & - & $\begin{array}{l}102.66 \\
25.57\end{array}$ \\
\hline \multirow{3}{*}{ January (1999) } & $\begin{array}{l}\text { Vaal Dam } \\
(\mathrm{n}=6)\end{array}$ & $\begin{array}{l}\text { Mean } \\
\text { Std. deviation }\end{array}$ & $\begin{array}{l}2.33 \\
1.64\end{array}$ & $\begin{array}{l}3.39 \\
1.80\end{array}$ & $\begin{array}{l}0.42 \\
0.27\end{array}$ & $\begin{array}{l}117.31 \\
42.69\end{array}$ & $\begin{array}{l}77.10 \\
21.35\end{array}$ & - \\
\hline & & p-value & ns & $<0.010$ & $<0.001$ & $<\mathbf{0 . 0 1 0}$ & $<0.001$ & - \\
\hline & $\begin{array}{l}\text { Barrage } \\
(\mathrm{n}=20)\end{array}$ & $\begin{array}{l}\text { Mean } \\
\text { Std. deviation }\end{array}$ & $\begin{array}{l}1.83 \\
1.32\end{array}$ & $\begin{array}{l}1.46 \\
1.30\end{array}$ & $\begin{array}{l}2.58 \\
1.59\end{array}$ & $\begin{array}{l}73.48 \\
24.27\end{array}$ & $\begin{array}{l}35.37 \\
13.76\end{array}$ & - \\
\hline \multirow{3}{*}{ March (1999) } & $\begin{array}{l}\text { Vaal Dam } \\
(\mathrm{n}=9)\end{array}$ & $\begin{array}{l}\text { Mean } \\
\text { Std. deviation }\end{array}$ & $\begin{array}{l}0.83 \\
0.93\end{array}$ & $\begin{array}{l}2.13 \\
1.98\end{array}$ & $\begin{array}{l}2.34 \\
1.10\end{array}$ & $\begin{array}{l}162.24 \\
28.08\end{array}$ & $\begin{array}{l}74.76 \\
12.56\end{array}$ & - \\
\hline & & p-value & ns & $<0.050$ & ns & $<0.001$ & $<0.001$ & - \\
\hline & $\begin{array}{l}\text { Barrage } \\
(\mathrm{n}=17)\end{array}$ & $\begin{array}{l}\text { Mean } \\
\text { Std. deviation }\end{array}$ & $\begin{array}{l}3.10 \\
3.24\end{array}$ & $\begin{array}{l}3.48 \\
1.21\end{array}$ & $\begin{array}{l}2.40 \\
1.59\end{array}$ & $\begin{array}{l}91.85 \\
15.99\end{array}$ & $\begin{array}{l}46.99 \\
8.57\end{array}$ & - \\
\hline \multirow{4}{*}{ June (1999) } & $\begin{array}{l}\text { Vaal Dam } \\
(\mathrm{n}=17)\end{array}$ & $\begin{array}{l}\text { Mean } \\
\text { Std. deviation }\end{array}$ & $\begin{array}{l}3.90 \\
3.17\end{array}$ & $\begin{array}{l}2.31 \\
1.28\end{array}$ & $\begin{array}{l}2.67 \\
1.44\end{array}$ & $\begin{array}{l}166.46 \\
20.55\end{array}$ & $\begin{array}{l}112.06 \\
34.02\end{array}$ & - \\
\hline & & p-value & ns & ns & ns & $<\mathbf{0 . 0 0 1}$ & $<0.001$ & - \\
\hline & Barrage & Mean & 3.67 & 1.18 & 3.74 & 71.86 & 32.00 & - \\
\hline & $(\mathrm{n}=6)$ & Std. deviation & 1.39 & 1.72 & 1.66 & 12.01 & 11.68 & - \\
\hline \multirow{3}{*}{ August (1999) } & $\begin{array}{l}\text { Vaal Dam } \\
(\mathrm{n}=18)\end{array}$ & $\begin{array}{l}\text { Mean } \\
\text { Std. deviation }\end{array}$ & $\begin{array}{l}2.25 \\
1.83\end{array}$ & $\begin{array}{l}1.59 \\
1.08\end{array}$ & $\begin{array}{l}3.43 \\
1.95\end{array}$ & $\begin{array}{l}149.93 \\
16.75\end{array}$ & $\begin{array}{l}111.49 \\
32.73\end{array}$ & - \\
\hline & & p-value & ns & ns & ns & $<0.001$ & $<0.001$ & - \\
\hline & $\begin{array}{l}\text { Barrage } \\
(\mathrm{n}=20)\end{array}$ & $\begin{array}{l}\text { Mean } \\
\text { Std. deviation }\end{array}$ & $\begin{array}{l}2.11 \\
2.12\end{array}$ & $\begin{array}{l}2.31 \\
1.41\end{array}$ & $\begin{array}{l}3.15 \\
1.76\end{array}$ & $\begin{array}{l}84.84 \\
10.05\end{array}$ & $\begin{array}{l}58.76 \\
17.11\end{array}$ & - \\
\hline \multirow{3}{*}{ October (1999) } & $\begin{array}{l}\text { Vaal Dam } \\
(\mathrm{n}=20)\end{array}$ & $\begin{array}{l}\text { Mean } \\
\text { Std. deviation }\end{array}$ & $\begin{array}{l}2.16 \\
1.27\end{array}$ & $\begin{array}{l}1.97 \\
1.90\end{array}$ & $\begin{array}{l}2.57 \\
2.67\end{array}$ & $\begin{array}{l}148.76 \\
23.46\end{array}$ & $\begin{array}{l}96.45 \\
32.01\end{array}$ & - \\
\hline & & p-value & $<\mathbf{0 . 0 5 0}$ & $<0.050$ & ns & $<0.001$ & $<0.001$ & - \\
\hline & $\begin{array}{l}\text { Barrage } \\
(\mathrm{n}=20)\end{array}$ & $\begin{array}{l}\text { Mean } \\
\text { Std. deviation }\end{array}$ & $\begin{array}{l}3.10 \\
1.10\end{array}$ & $\begin{array}{l}3.12 \\
1.54\end{array}$ & $\begin{array}{l}2.97 \\
0.89\end{array}$ & $\begin{array}{l}76.78 \\
16.99\end{array}$ & $\begin{array}{l}49.70 \\
15.86\end{array}$ & - \\
\hline \multirow{3}{*}{ February (2000) } & $\begin{array}{l}\text { Vaal Dam } \\
(\mathrm{n}=20)\end{array}$ & $\begin{array}{l}\text { Mean } \\
\text { Std. deviation }\end{array}$ & $\begin{array}{l}3.70 \\
2.50\end{array}$ & $\begin{array}{l}2.89 \\
2.37\end{array}$ & $\begin{array}{l}1.89 \\
1.42\end{array}$ & $\begin{array}{l}139.83 \\
28.34\end{array}$ & $\begin{array}{l}83.78 \\
17.48\end{array}$ & - \\
\hline & & p-value & ns & ns & ns & $<0.001$ & $<0.001$ & - \\
\hline & $\begin{array}{l}\text { Barrage } \\
(\mathrm{n}=17)\end{array}$ & $\begin{array}{l}\text { Mean } \\
\text { Std. deviation }\end{array}$ & $\begin{array}{l}2.79 \\
1.71\end{array}$ & $\begin{array}{l}3.70 \\
1.14\end{array}$ & $\begin{array}{l}1.81 \\
1.70\end{array}$ & $\begin{array}{l}63.82 \\
13.15\end{array}$ & $\begin{array}{l}39.41 \\
12.62\end{array}$ & $\begin{array}{l}- \\
-\end{array}$ \\
\hline \multirow{4}{*}{$\begin{array}{l}\text { Pooled for all } \\
\text { surveys }\end{array}$} & $\begin{array}{l}\text { Vaal Dam } \\
(\mathrm{n}=110)\end{array}$ & $\begin{array}{l}\text { Mean } \\
\text { Std. deviation }\end{array}$ & $\begin{array}{l}2.64 \\
2.22\end{array}$ & $\begin{array}{l}2.40 \\
1.92\end{array}$ & $\begin{array}{l}2.20 \\
1.89\end{array}$ & $\begin{array}{l}149.60 \\
27.46\end{array}$ & $\begin{array}{l}96.13 \\
30.62\end{array}$ & - \\
\hline & & p-value & ns & ns & ns & $<0.001$ & $<0.001$ & - \\
\hline & Barrage & Mean & 2.63 & 2.67 & 2.68 & 77.79 & 45.37 & - \\
\hline & $(n=100)$ & Std. deviation & 2.01 & 1.60 & 1.59 & 18.65 & 16.21 & - \\
\hline
\end{tabular}




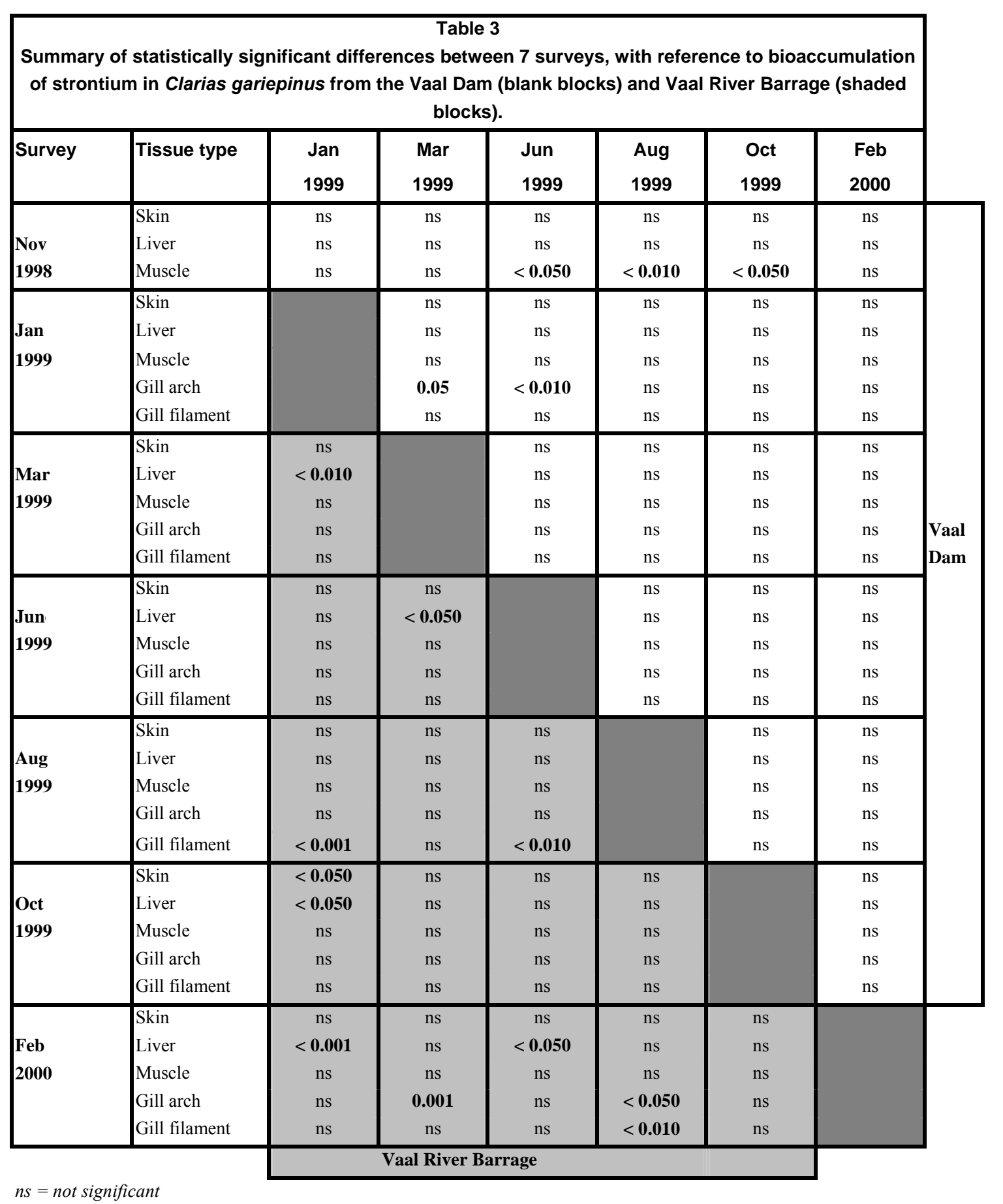

\section{Nickel}

\section{Water and sediment}

Concentrations recorded from tissues were generally higher than or equal to concentrations recorded in water, but lower than concentrations recorded in sediment. Tissues were ranked according to decreasing Al concentrations (Table 5) as follows: Vaal Dam - gill filament $>$ skin $>$ liver $\geq$ gill arch $>$ muscle; Vaal River Barrage - gill filament $>$ skin $\geq$ gill arch $>$ liver $>$ muscle. At both localities significantly higher Al concentrations in muscle were recorded during the June 1999 survey compared to other surveys (Table 6).
Lead

\section{Water and sediment}

All $\mathrm{Pb}$ concentrations were below the detection limit in both water $(0.1 \mathrm{mg} / \ell)$ and sediment $(0.05 \mathrm{mg} / \mathrm{g})$ respectively. The ranking of tissues and organs in terms of $\mathrm{Pb}$ concentration (Table 8) were: Vaal Dam - gill arch $\geq$ gill filament $>$ liver $\geq$ muscle $\geq$ skin; Vaal River Barrage - gill arch $>$ gill filament $\geq$ muscle $\geq$ liver $\geq$ skin. Seasonal trends in differences in metal tissue concentrations between localities were not evident (Table 9). 


\begin{tabular}{|c|c|c|c|}
\hline \multicolumn{4}{|c|}{$\begin{array}{c}\text { Table } 4 \\
\text { Aluminium concentrations in water and sediment of the } \\
\text { Vaal Dam and Vaal River Barrage, obtained during } \\
7 \text { surveys conducted between } 1998 \text { and } 2000\end{array}$} \\
\hline \multirow[t]{2}{*}{ Month of survey } & \multirow{2}{*}{$\begin{array}{l}\text { Water or sediment } \\
\text { sample }\end{array}$} & \multicolumn{2}{|c|}{ Locality } \\
\hline & & Vaal Dam & $\begin{array}{c}\text { Vaal River } \\
\text { Barrage }\end{array}$ \\
\hline \multirow{2}{*}{ November (1998) } & Water $(\mathrm{mg} / \ell)$ & 0.481 & N/A \\
\hline & Sediment (mg/g) & 41.657 & N/A \\
\hline \multirow[t]{2}{*}{ January (1999) } & Water $(\mathrm{mg} / \ell)$ & 0.101 & 0.361 \\
\hline & Sediment (mg/g) & 51.798 & 54.336 \\
\hline \multirow[t]{2}{*}{ March (1999) } & Water $(\mathrm{mg} / \ell)$ & 0.141 & $<0.050$ \\
\hline & Sediment (mg/g) & 52.787 & 51.819 \\
\hline \multirow[t]{2}{*}{ June (1999) } & Water $(\mathrm{mg} / \ell)$ & 0.161 & $<0.035$ \\
\hline & Sediment (mg/g) & 60.564 & 50.387 \\
\hline \multirow[t]{2}{*}{ August (1999) } & Water $(\mathrm{mg} / \ell)$ & $<0.035$ & $<0.035$ \\
\hline & Sediment (mg/g) & 35.751 & 51.017 \\
\hline \multirow[t]{2}{*}{ October (1999) } & Water $(\mathrm{mg} / \ell)$ & $<0.035$ & $<0.035$ \\
\hline & Sediment (mg/g) & 12.311 & 52.787 \\
\hline \multirow[t]{2}{*}{ February (2000) } & Water $(\mathrm{mg} / \ell)$ & $<0.035$ & $<0.035$ \\
\hline & Sediment (mg/g) & 33.386 & 60.541 \\
\hline \multicolumn{4}{|c|}{$\begin{array}{l}\text { Analyses by Department of Water Affairs and Forestry } \\
\text { (Institute for Water Quality Studies) } \\
\text { N/A = Not available }\end{array}$} \\
\hline \multicolumn{4}{|c|}{ Data provided by Rand Water Board } \\
\hline
\end{tabular}

\section{TABLE 5}

Aluminium concentrations ( $\mu \mathrm{g} / \mathrm{g}$ ) in 5 different tissue types of Clarias gariepinus from the Vaal Dam and Vaal River Barrage (shaded blocks). T-test p-values indicate whether concentrations recorded in each tissue type differed significantly between localities.

\begin{tabular}{|c|c|c|c|c|c|c|c|c|}
\hline \multirow[t]{2}{*}{ Month } & \multirow[t]{2}{*}{ Locality } & \multirow{2}{*}{$\begin{array}{l}\text { Statistical } \\
\text { variable }\end{array}$} & \multicolumn{6}{|c|}{ Tissue type } \\
\hline & & & Skin & Liver & Muscle & Gill arch & $\begin{array}{c}\text { Gill } \\
\text { filament }\end{array}$ & $\begin{array}{c}\text { Whole } \\
\text { gill }\end{array}$ \\
\hline \multirow[t]{2}{*}{ November (1998) } & Vaal Dam & Mean & 40.93 & 34.30 & 27.67 & - & - & 129.19 \\
\hline & $(n=20)$ & Std. deviation & 37.04 & 23.42 & 33.74 & - & - & 159.40 \\
\hline \multirow{5}{*}{ January (1999) } & Vaal Dam & Mean & 52.94 & 47.05 & 19.85 & 29.45 & 95.27 & - \\
\hline & $(\mathrm{n}=6)$ & Std. deviation & 36.86 & 30.24 & 22.02 & 34.09 & 52.42 & - \\
\hline & & p-value & ns & ns & ns & ns & ns & - \\
\hline & Barrage & Mean & 33.37 & 35.77 & 21.94 & 39.13 & 72.41 & - \\
\hline & $(n=20)$ & Std. deviation & 23.45 & 26.78 & 15.02 & 30.50 & 57.09 & - \\
\hline \multirow{5}{*}{ March (1999) } & Vaal Dam & Mean & 23.35 & 82.99 & 17.50 & 42.77 & 99.37 & - \\
\hline & $(\mathrm{n}=9)$ & Std. deviation & 13.14 & 61.93 & 14.65 & 18.35 & 83.42 & - \\
\hline & & p-value & ns & ns & $<0.050$ & ns & ns & - \\
\hline & Barrage & Mean & 23.74 & 48.58 & 32.93 & 42.08 & 162.57 & - \\
\hline & $(n=17)$ & Std. deviation & 18.69 & 20.34 & 17.56 & 27.47 & 84.63 & - \\
\hline \multirow{5}{*}{ June (1999) } & Vaal Dam & Mean & 128.58 & 55.85 & 57.69 & 74.02 & 112.94 & - \\
\hline & $(\mathrm{n}=17)$ & Std. deviation & 117.41 & 26.30 & 19.29 & 16.34 & 46.75 & - \\
\hline & & p-value & ns & $<0.050$ & ns & $<0.010$ & $<0.001$ & - \\
\hline & Barrage & Mean & 55.24 & 40.48 & 52.95 & 51.97 & 55.81 & - \\
\hline & $(n=6)$ & Std. deviation & 20.82 & 2.72 & 19.99 & 4.26 & 9.55 & - \\
\hline \multirow{5}{*}{ August (1999) } & Vaal Dam & Mean & 58.97 & 90.61 & 33.44 & 27.84 & 105.83 & - \\
\hline & $(\mathrm{n}=18)$ & Std. deviation & 92.44 & 159.09 & 12.36 & 29.89 & 75.65 & - \\
\hline & & p-value & ns & ns & ns & $<0.010$ & ns & - \\
\hline & Barrage & Mean & 48.97 & 40.20 & 33.96 & 53.48 & 65.57 & - \\
\hline & $(n=20)$ & Std. deviation & 11.59 & 18.51 & 15.63 & 7.33 & 32.07 & - \\
\hline
\end{tabular}


Table 5 (continued)

\begin{tabular}{|c|c|c|c|c|c|c|c|c|}
\hline \multirow{4}{*}{ October (1999) } & $\begin{array}{l}\text { Vaal Dam } \\
(\mathrm{n}=20)\end{array}$ & $\begin{array}{l}\text { Mean } \\
\text { Std. deviation }\end{array}$ & $\begin{array}{l}44.78 \\
34.35\end{array}$ & $\begin{array}{l}28.28 \\
25.31\end{array}$ & $\begin{array}{l}23.24 \\
26.03\end{array}$ & $\begin{array}{l}48.68 \\
15.25\end{array}$ & $\begin{array}{l}86.02 \\
33.85\end{array}$ & - \\
\hline & & p-value & ns & ns & ns & $<0.010$ & ns & - \\
\hline & Barrage & Mean & 53.47 & 24.62 & 34.66 & 35.78 & 53.92 & - \\
\hline & $(\mathrm{n}=20)$ & Std. deviation & 31.17 & 14.65 & 8.80 & 13.44 & 29.12 & - \\
\hline \multirow{5}{*}{ February (2000) } & Vaal Dam & Mean & 68.21 & 29.36 & 36.17 & 43.90 & 39.05 & - \\
\hline & $(\mathrm{n}=20)$ & Std. deviation & 73.11 & 25.64 & 23.97 & 25.72 & 30.81 & - \\
\hline & & p-value & ns & ns & $<0.050$ & ns & $<0.010$ & - \\
\hline & Barrage & Mean & 50.77 & 27.92 & 22.90 & 42.25 & 110.05 & - \\
\hline & $(\mathrm{n}=17)$ & Std. deviation & 35.88 & 12.37 & 9.50 & 18.58 & 93.26 & - \\
\hline \multirow{5}{*}{$\begin{array}{l}\text { Pooled for all } \\
\text { surveys }\end{array}$} & Vaal Dam & Mean & 62.30 & 49.53 & 32.73 & 46.36 & 94.21 & - \\
\hline & $(\mathrm{n}=110)$ & Std. deviation & 75.97 & 72.61 & 26.12 & 27.27 & 92.36 & - \\
\hline & & p-value & $<0.010$ & $<0.050$ & ns & $\mathrm{ns}$ & ns & - \\
\hline & Barrage & Mean & 43.14 & 36.08 & 30.78 & 43.13 & 88.07 & - \\
\hline & $(\mathrm{n}=100)$ & Std. deviation & 27.12 & 19.89 & 15.87 & 21.05 & 70.99 & - \\
\hline
\end{tabular}

\section{Table 6}

Summary of statistically significant differences between 7 surveys, with reference to bioaccumulation of aluminium in Clarias gariepinus from the Vaal Dam (blank blocks) and

\begin{tabular}{|c|c|c|c|c|c|c|c|}
\hline Survey & Tissue type & $\begin{array}{l}\text { Jan } \\
1999\end{array}$ & $\begin{array}{l}\text { Mar } \\
1999\end{array}$ & \begin{tabular}{|l} 
Jun \\
1999
\end{tabular} & $\begin{array}{l}\text { Aug } \\
1999\end{array}$ & $\begin{array}{l}\text { Oct } \\
1999\end{array}$ & \begin{tabular}{|l} 
Feb \\
2000
\end{tabular} \\
\hline $\begin{array}{l}\text { Nov } \\
1998\end{array}$ & \begin{tabular}{|l} 
Skin \\
Liver \\
Muscle
\end{tabular} & $\begin{array}{l}\text { ns } \\
\text { ns } \\
\text { ns }\end{array}$ & $\begin{array}{l}\text { ns } \\
\text { ns } \\
\text { ns }\end{array}$ & $\begin{array}{l}\text { ns } \\
\text { ns } \\
<\mathbf{0 . 0 5 0}\end{array}$ & $\begin{array}{l}\text { ns } \\
\text { ns } \\
\text { ns }\end{array}$ & $\begin{array}{l}\text { ns } \\
\text { ns } \\
\text { ns }\end{array}$ & $\begin{array}{l}\text { ns } \\
\text { ns } \\
\text { ns }\end{array}$ \\
\hline $\begin{array}{l}\text { Jan } \\
1999\end{array}$ & $\begin{array}{l}\text { Skin } \\
\text { Liver } \\
\text { Muscle } \\
\text { Gill arch } \\
\text { Gill filament }\end{array}$ & & $\begin{array}{l}\text { ns } \\
\text { ns } \\
\text { ns } \\
\text { ns } \\
\text { ns }\end{array}$ & $\begin{array}{l}\text { ns } \\
\text { ns } \\
<\mathbf{0 . 0 0 1} \\
\text { ns } \\
\text { ns }\end{array}$ & $\begin{array}{l}\text { ns } \\
\text { ns } \\
\text { ns } \\
\text { ns } \\
\text { ns }\end{array}$ & $\begin{array}{l}\text { ns } \\
\text { ns } \\
\text { ns } \\
\text { ns } \\
\text { ns }\end{array}$ & $\begin{array}{l}\text { ns } \\
\text { ns } \\
\text { ns } \\
\text { ns } \\
\text { ns }\end{array}$ \\
\hline $\begin{array}{l}\text { Mar } \\
1999\end{array}$ & $\begin{array}{l}\text { Skin } \\
\text { Liver } \\
\text { Muscle } \\
\text { Gill arch } \\
\text { Gill filament }\end{array}$ & $\begin{array}{l}\text { ns } \\
\text { ns } \\
\text { ns } \\
\text { ns } \\
<\mathbf{0 . 0 5 0}\end{array}$ & & $\begin{array}{l}<\mathbf{0 . 0 5 0} \\
\text { ns } \\
<\mathbf{0 . 0 0 1} \\
<\mathbf{0 . 0 1 0} \\
\text { ns }\end{array}$ & $\begin{array}{l}\text { ns } \\
\text { ns } \\
\text { ns } \\
\text { ns } \\
\text { ns }\end{array}$ & $\begin{array}{l}\text { ns } \\
\text { ns } \\
\text { ns } \\
\text { ns } \\
\text { ns }\end{array}$ & $\begin{array}{l}\text { ns } \\
\text { ns } \\
\text { ns } \\
\text { ns } \\
\text { ns }\end{array}$ \\
\hline $\begin{array}{l}\text { Jun } \\
1999\end{array}$ & $\begin{array}{l}\text { Skin } \\
\text { Liver } \\
\text { Muscle } \\
\text { Gill arch } \\
\text { Gill filament }\end{array}$ & $\begin{array}{l}\text { ns } \\
\text { ns } \\
\text { 0.001 } \\
\text { ns } \\
\text { ns } \\
\end{array}$ & $\begin{array}{l}\text { ns } \\
\text { ns } \\
\text { ns } \\
\text { ns } \\
\mathbf{0 . 0 0 1}\end{array}$ & & $\begin{array}{l}\text { ns } \\
\text { ns } \\
<\mathbf{0 . 0 0 1} \\
<\mathbf{0 . 0 0 1} \\
\text { ns }\end{array}$ & $\begin{array}{l}\text { ns } \\
\text { ns } \\
<\mathbf{0 . 0 0 1} \\
<\mathbf{0 . 0 0 1} \\
\text { ns }\end{array}$ & $\begin{array}{l}\text { ns } \\
\text { ns } \\
<\mathbf{0 . 0 0 1} \\
\text { ns } \\
\text { ns }\end{array}$ \\
\hline $\begin{array}{l}\text { Aug } \\
1999\end{array}$ & $\begin{array}{l}\text { Skin } \\
\text { Liver } \\
\text { Muscle } \\
\text { Gill arch } \\
\text { Gill filament }\end{array}$ & $\begin{array}{l}\text { ns } \\
\text { ns } \\
\text { ns } \\
\text { ns } \\
\text { ns }\end{array}$ & $\begin{array}{l}\text { 0.001 } \\
\text { ns } \\
\text { ns } \\
\text { ns } \\
\text { ns }\end{array}$ & $\begin{array}{l}\text { ns } \\
\text { ns } \\
\text { ns } \\
\text { ns } \\
\text { ns }\end{array}$ & & $\begin{array}{l}\text { ns } \\
\text { ns } \\
\text { ns } \\
\text { ns } \\
\text { ns }\end{array}$ & $\begin{array}{l}\text { ns } \\
\text { ns } \\
\text { ns } \\
\text { ns } \\
\text { ns }\end{array}$ \\
\hline $\begin{array}{l}\text { Oct } \\
1999\end{array}$ & $\begin{array}{l}\text { Skin } \\
\text { Liver } \\
\text { Muscle } \\
\text { Gill arch } \\
\text { Gill filament }\end{array}$ & $\begin{array}{l}\text { ns } \\
\text { ns } \\
\text { ns } \\
\text { ns } \\
\text { ns }\end{array}$ & $\begin{array}{l}<0.050 \\
<0.001 \\
\text { ns } \\
\text { ns } \\
0.001\end{array}$ & $\begin{array}{l}\text { ns } \\
<\mathbf{0 . 0 1 0} \\
\text { ns } \\
\mathbf{0 . 0 0 1} \\
\text { ns }\end{array}$ & $\begin{array}{l}\text { ns } \\
\text { ns } \\
\text { ns } \\
<\mathbf{0 . 0 0 1} \\
\text { ns }\end{array}$ & & $\begin{array}{l}\text { ns } \\
\text { ns } \\
\text { ns } \\
\text { ns } \\
\text { ns }\end{array}$ \\
\hline $\begin{array}{l}\text { Feb } \\
2000\end{array}$ & $\begin{array}{l}\text { Skin } \\
\text { Liver } \\
\text { Muscle } \\
\text { Gill arch } \\
\text { Gill filament } \\
\end{array}$ & $\begin{array}{l}\text { ns } \\
\text { ns } \\
\text { ns } \\
\text { ns } \\
\text { ns } \\
\end{array}$ & $\begin{array}{l}\text { ns } \\
\mathbf{0 . 0 0 1} \\
\text { ns } \\
\text { ns } \\
\text { ns } \\
\end{array}$ & $\begin{array}{l}\text { ns } \\
<\mathbf{0 . 0 5 0} \\
<\mathbf{0 . 0 1 0} \\
\text { ns } \\
\text { ns } \\
\end{array}$ & $\begin{array}{l}\text { ns } \\
\text { ns } \\
\text { ns } \\
\text { ns } \\
\text { ns }\end{array}$ & $\begin{array}{l}\text { ns } \\
\text { ns } \\
\text { ns } \\
\text { ns } \\
\text { ns } \\
\end{array}$ & \\
\hline
\end{tabular}




\begin{tabular}{|c|c|c|c|}
\hline \multicolumn{4}{|c|}{$\begin{array}{c}\text { Table } 7 \\
\text { Lead concentrations in water and sediment of the Vaal } \\
\text { Dam and Vaal River Barrage, obtained during } 7 \text { surveys } \\
\text { conducted between } 1998 \text { and } 2000\end{array}$} \\
\hline \multirow[t]{2}{*}{ Month of survey } & \multirow{2}{*}{$\begin{array}{l}\text { Water or sediment } \\
\text { sample }\end{array}$} & \multicolumn{2}{|c|}{ Locality } \\
\hline & & Vaal Dam & $\begin{array}{c}\text { Vaal River } \\
\text { Barrage }\end{array}$ \\
\hline \multirow[t]{2}{*}{ November (1998) } & \multirow{2}{*}{$\begin{array}{l}\text { Water }(\mathrm{mg} / \ell) \\
\text { Sediment }(\mathrm{mg} / \mathrm{g})\end{array}$} & $<0.100$ & N/A \\
\hline & & $<0.050$ & N/A \\
\hline \multirow[t]{2}{*}{ January (1999) } & \multirow{2}{*}{$\begin{array}{l}\text { Water }(\mathrm{mg} / \mathrm{l}) \\
\text { Sediment }(\mathrm{mg} / \mathrm{g})\end{array}$} & $<0.100$ & $<0.100$ \\
\hline & & $<0.050$ & $<0.050$ \\
\hline \multirow[t]{2}{*}{ March (1999) } & \multirow{2}{*}{$\begin{array}{l}\text { Water }(\mathrm{mg} / \ell) \\
\text { Sediment }(\mathrm{mg} / \mathrm{g})\end{array}$} & $<0.100$ & $<0.100$ \\
\hline & & $<0.050$ & $<0.050$ \\
\hline \multirow[t]{2}{*}{ June (1999) } & \multirow{2}{*}{$\begin{array}{l}\text { Water }(\mathrm{mg} / \ell) \\
\text { Sediment }(\mathrm{mg} / \mathrm{g})\end{array}$} & $<0.100$ & $<0.100$ \\
\hline & & $<0.050$ & $<0.050$ \\
\hline \multirow[t]{2}{*}{ August (1999) } & Water $(\mathrm{mg} / \ell)$ & $<0.100$ & $<0.100$ \\
\hline & Sediment (mg/g) & $<0.050$ & $<0.050$ \\
\hline \multirow[t]{2}{*}{ October (1999) } & Water $(\mathrm{mg} / \ell)$ & $<0.100$ & $<0.100$ \\
\hline & Sediment (mg/g) & $<0.050$ & $<0.050$ \\
\hline \multirow[t]{2}{*}{ February (2000) } & Water $(\mathrm{mg} / \ell)$ & $<0.100$ & $<0.100$ \\
\hline & Sediment (mg/g) & $<0.050$ & $<0.050$ \\
\hline \multicolumn{4}{|c|}{$\begin{array}{l}\text { Analyses by Department of Water Affairs and Forestry } \\
\text { (Institute for Water Quality Studies) }\end{array}$} \\
\hline \multicolumn{4}{|c|}{ Data provided by Rand Water Board } \\
\hline \multicolumn{4}{|c|}{$\begin{array}{l}\text { All concentrations are below the detection limit } \\
\text { N/A = Not available }\end{array}$} \\
\hline
\end{tabular}

\begin{tabular}{|c|c|c|c|c|c|c|c|c|}
\hline \multicolumn{9}{|c|}{$\begin{array}{c}\text { Table } 8 \\
\text { Lead concentrations ( } \mu \mathrm{g} / \mathrm{g} \text { ) in } 5 \text { different tissue types of Clarias gariepinus from the Vaal Dam and } \\
\text { Vaal River Barrage (shaded blocks). T-test } \mathrm{p} \text {-values indicate whether concentrations recorded } \\
\text { in each tissue type differed significantly between localities. }\end{array}$} \\
\hline \multirow[t]{2}{*}{ Month } & \multirow[t]{2}{*}{ Locality } & \multirow{2}{*}{$\begin{array}{l}\text { Statistical } \\
\text { variable }\end{array}$} & \multicolumn{4}{|c|}{ Tissue type } & \multirow[b]{2}{*}{\begin{tabular}{|l} 
Gill \\
filament
\end{tabular}} & \multirow[b]{2}{*}{\begin{tabular}{|l} 
Whole \\
gill
\end{tabular}} \\
\hline & & & Skin & Liver & Muscle & Gill arch & & \\
\hline November (1998) & $\begin{array}{l}\text { Vaal Dam } \\
(\mathrm{n}=20)\end{array}$ & \begin{tabular}{|l|} 
Mean \\
Std. deviation
\end{tabular} & \begin{tabular}{|l|}
1.14 \\
1.09
\end{tabular} & $\begin{array}{l}10.37 \\
2.85\end{array}$ & $\begin{array}{l}0.59 \\
0.67\end{array}$ & - & - & \begin{tabular}{|l|}
10.29 \\
8.23
\end{tabular} \\
\hline \multirow{3}{*}{ January (1999) } & $\begin{array}{l}\text { Vaal Dam } \\
(\mathrm{n}=6)\end{array}$ & \begin{tabular}{|l} 
Mean \\
Std. deviation
\end{tabular} & $\begin{array}{l}9.21 \\
2.12 \\
\end{array}$ & $\begin{array}{l}2.57 \\
1.73 \\
\end{array}$ & \begin{tabular}{|l|}
5.67 \\
0.87 \\
\end{tabular} & \begin{tabular}{|l|}
8.90 \\
5.96 \\
\end{tabular} & $\begin{array}{l}14.82 \\
3.23 \\
\end{array}$ & - \\
\hline & & p-value & $<0.001$ & $<<0.050$ & ns & ns & ns & - \\
\hline & $\begin{array}{l}\text { Barrage } \\
(\mathrm{n}=20)\end{array}$ & $\begin{array}{l}\text { Mean } \\
\text { Std. deviation }\end{array}$ & $\begin{array}{l}2.26 \\
1.84\end{array}$ & \begin{tabular}{|l|}
5.51 \\
2.67
\end{tabular} & $\begin{array}{l}5.23 \\
2.15\end{array}$ & $\begin{array}{l}12.93 \\
3.78 \\
\end{array}$ & $\begin{array}{l}14.06 \\
5.11\end{array}$ & - \\
\hline \multirow{3}{*}{ March (1999) } & $\begin{array}{l}\text { Vaal Dam } \\
(\mathrm{n}=9)\end{array}$ & \begin{tabular}{|l} 
Mean \\
Std. deviation
\end{tabular} & \begin{tabular}{|l|}
4.79 \\
2.97 \\
\end{tabular} & \begin{tabular}{|l|}
3.34 \\
2.18 \\
\end{tabular} & \begin{tabular}{|l|}
2.64 \\
2.28 \\
\end{tabular} & \begin{tabular}{|l|}
15.02 \\
7.09 \\
\end{tabular} & \begin{tabular}{|l|}
12.48 \\
6.84 \\
\end{tabular} & - \\
\hline & & p-value & ns & ns & $<<0.010$ & ns & ns & - \\
\hline & $\begin{array}{l}\text { Barrage } \\
(\mathrm{n}=17)\end{array}$ & $\begin{array}{l}\text { Mean } \\
\text { Std. deviation }\end{array}$ & $\begin{array}{l}4.15 \\
2.35\end{array}$ & $\begin{array}{l}2.96 \\
3.15\end{array}$ & $\begin{array}{l}5.52 \\
1.91\end{array}$ & \begin{tabular}{|l|}
14.79 \\
2.23
\end{tabular} & \begin{tabular}{|l|}
8.75 \\
2.53
\end{tabular} & - \\
\hline \multirow{3}{*}{ June (1999) } & $\begin{array}{l}\text { Vaal Dam } \\
(\mathrm{n}=17)\end{array}$ & \begin{tabular}{|l} 
Mean \\
Std. deviation
\end{tabular} & \begin{tabular}{|l|}
3.51 \\
1.64 \\
\end{tabular} & \begin{tabular}{|l|}
4.92 \\
3.25 \\
\end{tabular} & \begin{tabular}{|l|}
4.29 \\
3.46 \\
\end{tabular} & \begin{tabular}{|l|}
14.77 \\
1.38 \\
\end{tabular} & \begin{tabular}{|l|}
10.74 \\
2.76 \\
\end{tabular} & $\begin{array}{l}- \\
-\end{array}$ \\
\hline & & p-value & ns & ns & ns & ns & ns & - \\
\hline & \begin{tabular}{|l} 
Barrage \\
$(\mathrm{n}=6)$
\end{tabular} & \begin{tabular}{|l} 
Mean \\
Std. deviation
\end{tabular} & $\begin{array}{l}2.90 \\
1.13 \\
\end{array}$ & \begin{tabular}{|l|}
2.27 \\
0.82 \\
\end{tabular} & \begin{tabular}{|l|}
4.02 \\
0.89
\end{tabular} & \begin{tabular}{|l|}
14.74 \\
1.02 \\
\end{tabular} & \begin{tabular}{|l|}
12.42 \\
2.63 \\
\end{tabular} & - \\
\hline \multirow{3}{*}{ August (1999) } & $\begin{array}{l}\text { Vaal Dam } \\
(\mathrm{n}=18)\end{array}$ & \begin{tabular}{|l|} 
Mean \\
Std. deviation
\end{tabular} & \begin{tabular}{|l|}
2.61 \\
0.73
\end{tabular} & $\begin{array}{l}2.33 \\
0.87\end{array}$ & \begin{tabular}{|l|}
2.26 \\
0.64
\end{tabular} & \begin{tabular}{|l|}
12.34 \\
1.56
\end{tabular} & $\begin{array}{l}9.65 \\
2.73\end{array}$ & $\begin{array}{l}- \\
-\end{array}$ \\
\hline & & p-value & ns & ns & $<0.001$ & 0.001 & ns & - \\
\hline & $\begin{array}{l}\text { Barrage } \\
(n=20)\end{array}$ & $\begin{array}{l}\text { Mean } \\
\text { Std. deviation }\end{array}$ & $\begin{array}{l}2.53 \\
1.38\end{array}$ & $\begin{array}{l}1.92 \\
1.32\end{array}$ & $\begin{array}{l}3.67 \\
0.80\end{array}$ & \begin{tabular}{|l|}
14.00 \\
1.17
\end{tabular} & \begin{tabular}{|l|}
8.19 \\
2.40
\end{tabular} & $\begin{array}{l}- \\
-\end{array}$ \\
\hline
\end{tabular}


Table 8 (continued)

\begin{tabular}{|c|c|c|c|c|c|c|c|c|}
\hline \multirow{4}{*}{ October (1999) } & $\begin{array}{l}\text { Vaal Dam } \\
(\mathrm{n}=20)\end{array}$ & $\begin{array}{l}\text { Mean } \\
\text { Std. deviation }\end{array}$ & $\begin{array}{l}2.19 \\
0.68\end{array}$ & $\begin{array}{l}3.14 \\
0.64\end{array}$ & $\begin{array}{l}3.53 \\
0.82\end{array}$ & $\begin{array}{l}12.76 \\
1.54\end{array}$ & $\begin{array}{l}9.69 \\
1.74\end{array}$ & $\begin{array}{l}- \\
-\end{array}$ \\
\hline & & p-value & 0.001 & $<0.010$ & ns & $<0.050$ & $<0.050$ & - \\
\hline & Barrage & Mean & 3.97 & 4.17 & 3.87 & 11.63 & 8.25 & - \\
\hline & $(n=20)$ & Std. deviation & 1.94 & 1.16 & 4.57 & 1.66 & 2.00 & - \\
\hline \multirow{5}{*}{ February (2000) } & Vaal Dam & Mean & 2.29 & 2.37 & 4.09 & 11.85 & 8.85 & - \\
\hline & $(\mathrm{n}=20)$ & Std. deviation & 0.41 & 1.36 & 1.08 & 2.10 & 1.65 & - \\
\hline & & p-value & $<0.001$ & ns & $<0.001$ & ns & ns & - \\
\hline & Barrage & Mean & 3.43 & 2.81 & 2.75 & 11.53 & 8.02 & - \\
\hline & $(\mathrm{n}=17)$ & Std. deviation & 0.52 & 0.39 & 0.45 & 1.55 & 1.42 & - \\
\hline \multirow{5}{*}{$\begin{array}{l}\text { Pooled for all } \\
\text { surveys }\end{array}$} & Vaal Dam & Mean & 2.89 & 4.44 & 3.05 & 12.82 & 10.32 & - \\
\hline & $(\mathrm{n}=110)$ & Std. deviation & 2.22 & 3.55 & 2.17 & 3.36 & 3.37 & - \\
\hline & & p-value & ns & ns & ns & ns & ns & - \\
\hline & Barrage & Mean & 3.21 & 3.44 & 4.20 & 13.07 & 9.69 & - \\
\hline & $(n=100)$ & Std. deviation & 1.81 & 2.29 & 2.58 & 2.54 & 3.80 & - \\
\hline
\end{tabular}

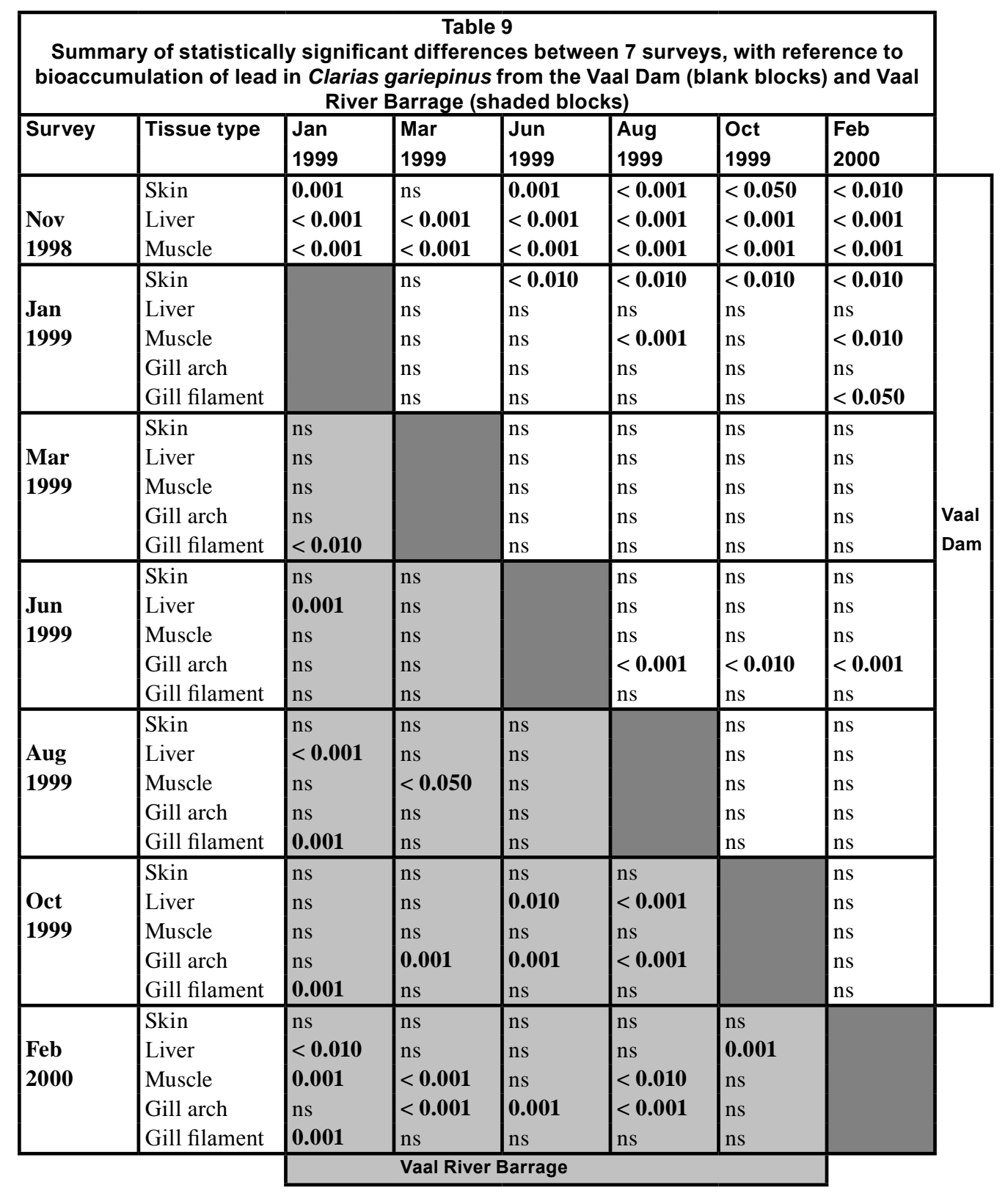




\begin{tabular}{|c|c|c|c|}
\hline \multicolumn{4}{|c|}{$\begin{array}{l}\text { Table } 10 \\
\text { Nickel concentrations in water and sediment of the Vaal Dam } \\
\text { and Vaal River Barrage, obtained during } 7 \text { surveys conducted } \\
\text { between } 1998 \text { and } 2000\end{array}$} \\
\hline \multirow[t]{2}{*}{ Month of survey } & \multirow{2}{*}{$\begin{array}{l}\text { Water or sediment } \\
\text { sample }\end{array}$} & \multicolumn{2}{|c|}{ Locality } \\
\hline & & Vaal Dam & $\begin{array}{c}\text { Vaal River } \\
\text { Barrage }\end{array}$ \\
\hline \multirow[t]{2}{*}{ November (1998) } & \multirow{2}{*}{$\begin{array}{l}\text { Water }(\mathrm{mg} / \ell) \\
\text { Sediment }(\mathrm{mg} / \mathrm{g})\end{array}$} & $<0.050$ & N/A \\
\hline & & $<0.009$ & N/A \\
\hline \multirow[t]{2}{*}{ January (1999) } & \multirow{2}{*}{$\begin{array}{l}\text { Water }(\mathrm{mg} / \ell) \\
\text { Sediment }(\mathrm{mg} / \mathrm{g})\end{array}$} & $<0.050$ & $<0.050$ \\
\hline & & $<0.009$ & $<0.009$ \\
\hline \multirow[t]{2}{*}{ March (1999) } & \multirow{2}{*}{$\begin{array}{l}\text { Water }(\mathrm{mg} / \ell) \\
\text { Sediment }(\mathrm{mg} / \mathrm{g})\end{array}$} & $<0.050$ & $<0.050$ \\
\hline & & $<0.009$ & $<0.009$ \\
\hline \multirow[t]{2}{*}{ June (1999) } & \multirow{2}{*}{$\begin{array}{l}\text { Water }(\mathrm{mg} / \ell) \\
\text { Sediment }(\mathrm{mg} / \mathrm{g})\end{array}$} & $<0.050$ & $<0.011$ \\
\hline & & $<0.009$ & $<0.009$ \\
\hline \multirow[t]{2}{*}{ August (1999) } & Water $(\mathrm{mg} / \ell)$ & $<0.011$ & $<0.011$ \\
\hline & Sediment (mg/g) & $<0.009$ & $<0.009$ \\
\hline \multirow[t]{2}{*}{ October (1999) } & Water (mg/l) & $<0.011$ & $<0.011$ \\
\hline & Sediment (mg/g) & $<0.009$ & 0.012 \\
\hline \multirow[t]{2}{*}{ February (2000) } & Water $(\mathrm{mg} / \ell)$ & $<0.011$ & $<0.011$ \\
\hline & Sediment (mg/g) & $<0.009$ & $<0.009$ \\
\hline \multicolumn{4}{|c|}{$\begin{array}{l}\text { Analyses by Department of Water Affairs } \\
\text { (Institute for Water Quality Studies) }\end{array}$} \\
\hline \multicolumn{4}{|c|}{ Data provided by Rand Water Board } \\
\hline \multicolumn{4}{|l|}{ N/A $=$ Not available } \\
\hline
\end{tabular}

Nickel concentrations in both water and sediment were below the limits for detection (50 and $11 \mu \mathrm{g} / \ell$, and $9 \mu \mathrm{g} / \mathrm{g}$ respectively). The low concentrations in sediment and water were mirrored by low Ni concentrations in C. gariepinus tissues. Concentrations recorded in the different tissues (Table 11), listed in decreasing order, were: Vaal Dam - gill arch $\geq$ gill filament $\geq$ muscle $\geq$ liver $\geq$ skin; Vaal River Barrage - gill arch $\geq$ skin $\geq$ muscle $\geq$ gill filament $\geq$ liver. With regards to significant seasonal differences within the localities no clear trends emerged (Table 12).

\section{Factors that may influence metal availability}

Readings and concentrations of 6 variables that may influence metal availability are given in Fig. 1 (next page). Peak flow readings in $\mathrm{m}^{3} / \mathrm{s}$ were obtained from the Department of Water Affairs database (recorded from the De Vaal Schoemansdrift gauging weir, station number C2H018-A01).

\section{Summary of results}

\section{Water and sediment}

Some of the readings, as indicated in the tables listed earlier, have been obtained from Rand Water Board and others from the Department of Water Affairs. As a result, 2 detection limits have been recorded for the same metal in many cases. Please note that this may give rise to 'false' trends.

Concentrations of the 4 metals under review in sediment and water can be divided into 3 classes. For $\mathrm{Pb}$ and $\mathrm{Ni}$ very low concentrations (mostly below the detection limits) were recorded in both sediment and water at both localities. Strontium concentrations were recorded in both sediment and water, with no readings under the detection limit being recorded. For Al high concentrations were recorded from sediment, while most water concentrations were found to be below detection. The high $\mathrm{Al}$ concentrations recorded from the Vaal River Barrage water samples during the October 1999 and January 1999 surveys were possibly the result of effluent or re-suspension from sediment. Jackson et al. (2009) also recorded high Al concentrations in water and sediment from the Plankenburg and Diep rivers (Western Cape). They could also not identify point sources of pollution.

In general, metal concentrations were not much higher at any one locality, as is evident from the similar maximum concentrations recorded at both localities.

\section{Concentrations in fish tissue}

Significant seasonal differences in concentration were recorded for all metals, but the same tissues did not always indicate these differences. In terms of significant $(p<0.05)$ differences in metal concentrations in fish tissue recorded from the 2 localities (Table 13), some general trends do emerge.

Strontium concentrations in gill tissue were significantly higher at the Vaal Dam during all individual surveys and when pooled for surveys. A similar trend was observed with regards to $\mathrm{Ni}$ concentration. For $\mathrm{Al}, \mathrm{Pb}$ and, to a lesser extent, $\mathrm{Ni}$, many of the differences between localities related to concentrations in muscle, skin and liver. When considering the number of 'month classes' for which significant differences were detected, concentrations of $\mathrm{Sr}$ and $\mathrm{Al}$ were higher more often at the Vaal 


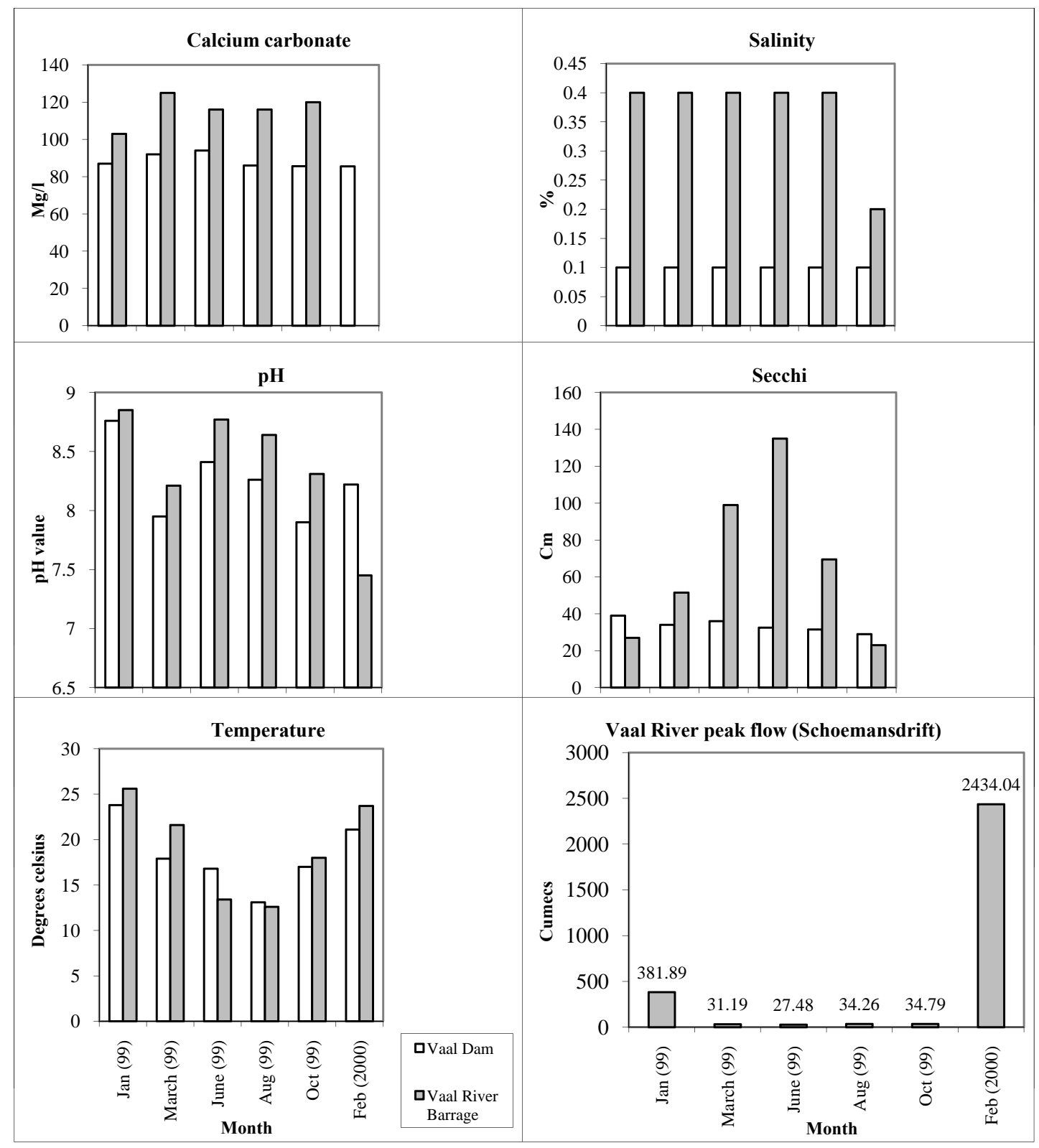

Figure 1

Concentrations and values for variables that affect metal toxicity and availability: water hardness (as calcium carbonate), salinity, $\mathrm{pH}$, suspended solid particles (represented by Secchi disk readings), temperature and peak flow

Dam while $\mathrm{Pb}$ and Ni were higher more often at the Vaal River Barrage.

Ranking tissues on the grounds of metal concentrations also allows identification of some trends. At both the Vaal Dam and Vaal River Barrage gill filament tissue was often ranked second with regard to metals concentrations encountered. The highest metal concentrations were most often recorded in gill arch, gill filament or liver tissue, with gill arch being ranked first most often. At both localities the lowest metal concentrations were most often recorded in muscle and skin tissue.

\section{Discussion}

The Vaal River Barrage receives domestic and industrial runoff and effluent from the PWV area, through streams like the Rietspruit and the Klip and Suikerbosrand Rivers. In terms of physical variables (e.g. salinity and conductivity) and macrodeterminants (phosphates, sulphates etc.), water quality at the Vaal River Barrage is poorer when compared to the Vaal Dam (Crafford and Avenant-Oldewage, 2009). However, contrary to expectations, metal concentrations in sediment and water recorded at both localities were similar. Braune and Rogers (1987) stated that the Vaal River catchment could be divided into 4 zones on the basis of water quality. According to their classification water quality in the Vaal Dam subcatchment is better than water quality in the Vaal River Barrage subcatchment. In view of the trace metal analyses results this is not necessarily the case. They do however continue by saying that 'this deterioration is fortunately in line with the general distribution of user water quality requirements in the catchment'. They state that water use in the Vaal Dam subcatchment is geared towards power stations and urban use (i.e. drinking water). From this 
Table 11

Nickel concentrations $(\mu \mathrm{g} / \mathrm{g}$ ) in 5 different tissue types of Clarias gariepinus from the Vaal Dam and Vaal River Barrage (shaded blocks). T-test $p$-values indicate whether concentrations recorded in each tissue type differed significantly between localities.

\begin{tabular}{|c|c|c|c|c|c|c|c|c|}
\hline \multirow[t]{2}{*}{ Month } & \multirow[t]{2}{*}{ Locality } & \multirow{2}{*}{$\begin{array}{l}\text { Statistical } \\
\text { variable }\end{array}$} & \multirow[b]{2}{*}{ Skin } & \multirow[b]{2}{*}{ Liver } & \multicolumn{3}{|c|}{ Tissue type } & \multirow[b]{2}{*}{$\begin{array}{l}\text { Whole } \\
\text { gill }\end{array}$} \\
\hline & & & & & Muscle & Gill arch & $\begin{array}{l}\text { Gill } \\
\text { filament }\end{array}$ & \\
\hline November (1998) & $\begin{array}{l}\text { Vaal Dam } \\
(\mathrm{n}=20)\end{array}$ & $\begin{array}{l}\text { Mean } \\
\text { Std. deviation }\end{array}$ & $\begin{array}{l}2.49 \\
1.25\end{array}$ & $\begin{array}{l}0.66 \\
1.33\end{array}$ & $\begin{array}{l}2.02 \\
0.55\end{array}$ & $\begin{array}{l}- \\
-\end{array}$ & - & $\begin{array}{l}6.58 \\
1.22\end{array}$ \\
\hline \multirow{3}{*}{ January (1999) } & $\begin{array}{l}\text { Vaal Dam } \\
(\mathrm{n}=6)\end{array}$ & $\begin{array}{l}\text { Mean } \\
\text { Std. deviation }\end{array}$ & $\begin{array}{l}2.08 \\
1.02\end{array}$ & $\begin{array}{l}1.18 \\
1.76\end{array}$ & $\begin{array}{l}0.36 \\
0.54\end{array}$ & $\begin{array}{l}2.06 \\
2.68\end{array}$ & $\begin{array}{l}2.97 \\
3.13\end{array}$ & $\begin{array}{l}- \\
-\end{array}$ \\
\hline & & p-value & ns & ns & $<0.050$ & ns & ns & - \\
\hline & $\begin{array}{l}\text { Barrage } \\
(\mathrm{n}=20)\end{array}$ & $\begin{array}{l}\text { Mean } \\
\text { Std. deviation }\end{array}$ & $\begin{array}{l}3.51 \\
1.92\end{array}$ & $\begin{array}{l}0.09 \\
0.27\end{array}$ & $\begin{array}{l}1.41 \\
1.55\end{array}$ & $\begin{array}{l}3.98 \\
2.41\end{array}$ & $\begin{array}{l}0.81 \\
1.14\end{array}$ & $\begin{array}{l}- \\
-\end{array}$ \\
\hline \multirow{3}{*}{ March (1999) } & $\begin{array}{l}\text { Vaal Dam } \\
(\mathrm{n}=9)\end{array}$ & $\begin{array}{l}\text { Mean } \\
\text { Std. deviation }\end{array}$ & $\begin{array}{l}0 \\
0\end{array}$ & $\begin{array}{l}0.20 \\
0.33\end{array}$ & $\begin{array}{l}8.51 \\
3.30\end{array}$ & $\begin{array}{l}1.07 \\
2.12\end{array}$ & $\begin{array}{l}4.17 \\
3.92\end{array}$ & $\begin{array}{l}- \\
-\end{array}$ \\
\hline & & p-value & 0.001 & ns & $<0.050$ & ns & $<0.050$ & - \\
\hline & $\begin{array}{l}\text { Barrage } \\
(n=17)\end{array}$ & $\begin{array}{l}\text { Mean } \\
\text { Std. deviation }\end{array}$ & $\begin{array}{l}4.33 \\
4.64\end{array}$ & $\begin{array}{l}0.52 \\
0.71\end{array}$ & $\begin{array}{l}4.97 \\
4.75\end{array}$ & $\begin{array}{l}1.42 \\
1.75\end{array}$ & $\begin{array}{l}1.34 \\
1.45\end{array}$ & $\begin{array}{l}- \\
-\end{array}$ \\
\hline \multirow{3}{*}{ June (1999) } & $\begin{array}{l}\text { Vaal Dam } \\
(\mathrm{n}=17)\end{array}$ & $\begin{array}{l}\text { Mean } \\
\text { Std. deviation }\end{array}$ & $\begin{array}{l}2.39 \\
0.83\end{array}$ & $\begin{array}{l}1.89 \\
0.73\end{array}$ & $\begin{array}{l}2.57 \\
1.50\end{array}$ & $\begin{array}{l}4.62 \\
1.19\end{array}$ & $\begin{array}{l}0.73 \\
0.62\end{array}$ & $\begin{array}{l}- \\
-\end{array}$ \\
\hline & & p-value & $<\mathbf{0 . 0 1 0}$ & ns & $<0.050$ & ns & $<\mathbf{0 . 0 0 1}$ & - \\
\hline & $\begin{array}{l}\text { Barrage } \\
(\mathrm{n}=6)\end{array}$ & $\begin{array}{l}\text { Mean } \\
\text { Std. deviation }\end{array}$ & $\begin{array}{l}1.12 \\
0.57\end{array}$ & $\begin{array}{l}1.55 \\
0.26\end{array}$ & $\begin{array}{l}1.14 \\
0.63\end{array}$ & $\begin{array}{l}4.30 \\
0.55\end{array}$ & $\begin{array}{l}4.72 \\
0.75\end{array}$ & - \\
\hline \multirow{3}{*}{ August (1999) } & $\begin{array}{l}\text { Vaal Dam } \\
(\mathrm{n}=18)\end{array}$ & $\begin{array}{l}\text { Mean } \\
\text { Std. deviation }\end{array}$ & $\begin{array}{l}1.14 \\
0.37\end{array}$ & $\begin{array}{l}1.21 \\
0.38\end{array}$ & $\begin{array}{l}0.46 \\
0.30\end{array}$ & $\begin{array}{l}8.56 \\
1.12\end{array}$ & $\begin{array}{l}5.00 \\
1.17\end{array}$ & $\begin{array}{l}- \\
-\end{array}$ \\
\hline & & p-value & ns & $<0.001$ & $<0.001$ & $<0.001$ & $<0.001$ & - \\
\hline & $\begin{array}{l}\text { Barrage } \\
(\mathrm{n}=20)\end{array}$ & $\begin{array}{l}\text { Mean } \\
\text { Std. deviation }\end{array}$ & $\begin{array}{l}1.12 \\
0.47\end{array}$ & $\begin{array}{l}2.09 \\
0.59 \\
\end{array}$ & $\begin{array}{l}1.47 \\
0.53\end{array}$ & $\begin{array}{l}4.43 \\
0.55 \\
\end{array}$ & $\begin{array}{l}2.99 \\
0.70\end{array}$ & $\begin{array}{l}- \\
-\end{array}$ \\
\hline \multirow{3}{*}{ October (1999) } & $\begin{array}{l}\text { Vaal Dam } \\
(\mathrm{n}=20)\end{array}$ & $\begin{array}{l}\text { Mean } \\
\text { Std. deviation }\end{array}$ & $\begin{array}{l}0.20 \\
0.39\end{array}$ & $\begin{array}{l}2.26 \\
1.18\end{array}$ & $\begin{array}{l}1.38 \\
0.85\end{array}$ & $\begin{array}{l}8.40 \\
4.03\end{array}$ & $\begin{array}{l}4.47 \\
1.89\end{array}$ & $\begin{array}{l}- \\
-\end{array}$ \\
\hline & & p-value & $<\mathbf{0 . 0 1 0}$ & ns & 0.001 & $<0.001$ & $<\mathbf{0 . 0 1 0}$ & - \\
\hline & $\begin{array}{l}\text { Barrage } \\
(\mathrm{n}=20)\end{array}$ & $\begin{array}{l}\text { Mean } \\
\text { Std. deviation }\end{array}$ & $\begin{array}{l}0.87 \\
0.92\end{array}$ & $\begin{array}{l}2.86 \\
1.23\end{array}$ & $\begin{array}{l}3.20 \\
2.05\end{array}$ & $\begin{array}{l}2.36 \\
3.02\end{array}$ & $\begin{array}{l}2.91 \\
1.54\end{array}$ & - \\
\hline \multirow{3}{*}{ February (2000) } & $\begin{array}{l}\text { Vaal Dam } \\
(\mathrm{n}=20)\end{array}$ & $\begin{array}{l}\text { Mean } \\
\text { Std. deviation }\end{array}$ & $\begin{array}{l}0.00 \\
0.00\end{array}$ & $\begin{array}{l}2.44 \\
2.22\end{array}$ & $\begin{array}{l}0.58 \\
0.69\end{array}$ & $\begin{array}{l}4.79 \\
2.59\end{array}$ & $\begin{array}{l}3.39 \\
2.76\end{array}$ & $\begin{array}{l}- \\
-\end{array}$ \\
\hline & & p-value & $<\mathbf{0 . 0 1 0}$ & ns & ns & $<0.001$ & ns & - \\
\hline & $\begin{array}{l}\text { Barrage } \\
(\mathrm{n}=17)\end{array}$ & $\begin{array}{l}\text { Mean } \\
\text { Std. deviation }\end{array}$ & $\begin{array}{l}4.18 \\
4.79 \\
\end{array}$ & $\begin{array}{l}2.97 \\
1.07 \\
\end{array}$ & $\begin{array}{l}2.04 \\
3.04\end{array}$ & $\begin{array}{l}1.57 \\
0.98\end{array}$ & $\begin{array}{l}2.28 \\
0.95 \\
\end{array}$ & $\begin{array}{l}- \\
-\end{array}$ \\
\hline \multirow{3}{*}{$\begin{array}{l}\text { Pooled for all } \\
\text { surveys }\end{array}$} & $\begin{array}{l}\text { Vaal Dam } \\
(\mathrm{n}=110)\end{array}$ & $\begin{array}{l}\text { Mean } \\
\text { Std. deviation }\end{array}$ & $\begin{array}{l}1.16 \\
1.27\end{array}$ & $\begin{array}{l}1.55 \\
1.50\end{array}$ & $\begin{array}{l}1.91 \\
2.44\end{array}$ & $\begin{array}{l}5.76 \\
3.60\end{array}$ & $\begin{array}{l}3.50 \\
2.62\end{array}$ & $\begin{array}{l}- \\
-\end{array}$ \\
\hline & & p-value & $<0.001$ & ns & ns & $<0.001$ & $<0.001$ & - \\
\hline & $\begin{array}{l}\text { Barrage } \\
(n=100)\end{array}$ & $\begin{array}{l}\text { Mean } \\
\text { Std. deviation }\end{array}$ & $\begin{array}{l}2.61 \\
3.23\end{array}$ & $\begin{array}{l}1.69 \\
1.41\end{array}$ & $\begin{array}{l}2.48 \\
2.87\end{array}$ & $\begin{array}{l}2.92 \\
2.27\end{array}$ & $\begin{array}{l}2.24 \\
1.56\end{array}$ & - \\
\hline
\end{tabular}

it is concluded that 'water quality', as the term is used by Braune and Rogers (1987), refers to the physical properties of water that determines its acceptability for human consumption, rather than metal pollution. This assumption is confirmed when Braune and Rogers (1987) discuss water quality problems in the Vaal River Barrage subcatchments. They only refer to eutrophication (resulting from organic pollution) and salinity. Organic pollution in the Vaal River Barrage thus appears to take precedence over trace metal pollution. In future, if authors refer to these water quality zones, mention should be made of the specific type(s) of water quality problems addressed. In this respect it may be worthwhile to conduct studies in the 2 lower subcatchments (Bloemhof and Douglas weir subcatchments) to determine the prevalence of heavy metal pollution.

Several possible explanations exist for the fact that significantly higher concentrations of metals were not consistently recorded in tissues from the Vaal River Barrage. In view of sediment and water metal concentrations, the simplest explanation would be that the biologically available metal concentrations at the 2 localities were similar. This is also the most likely 


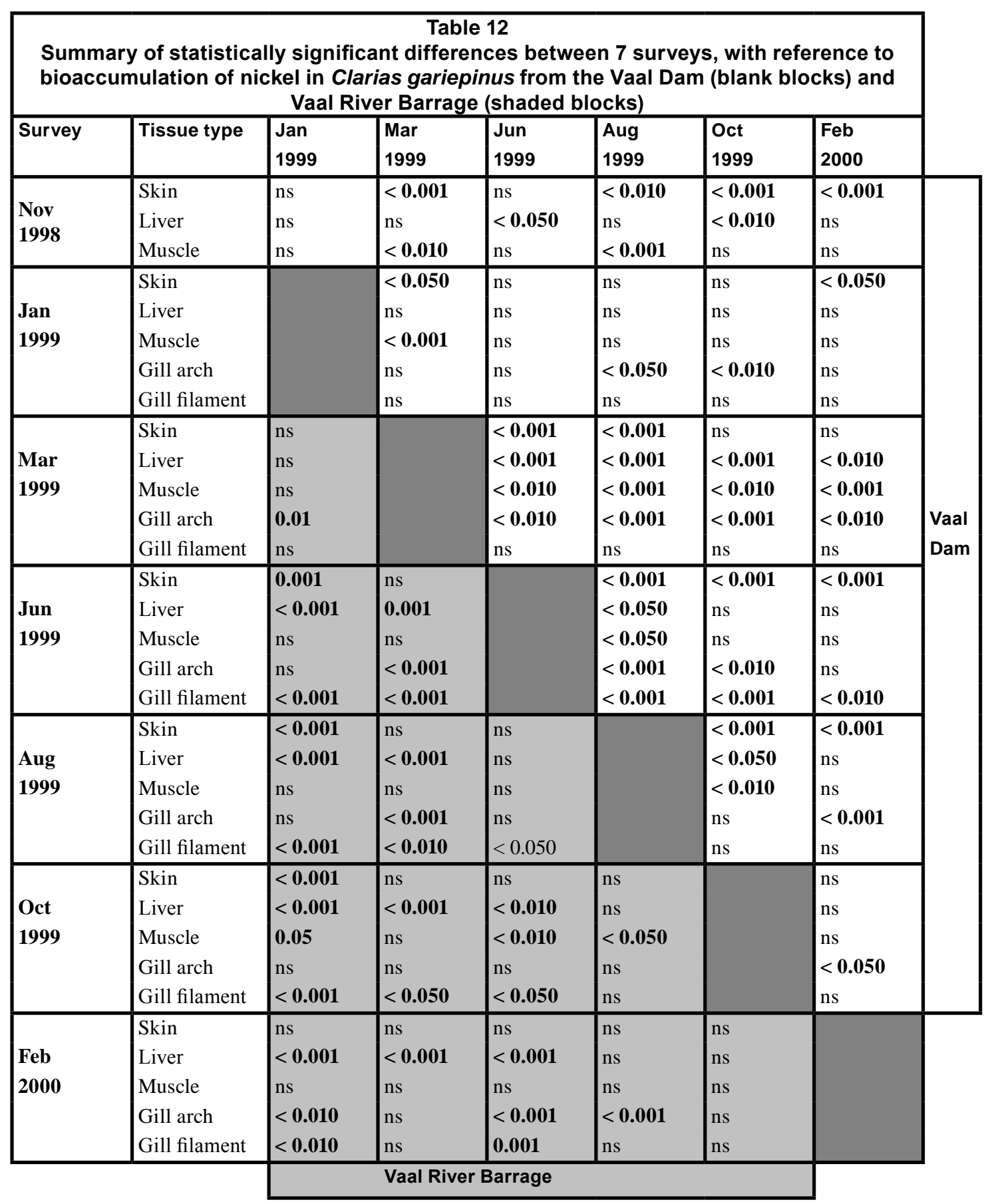

explanation. However, Clements and Rees (1997) warn that bioaccumulation of metals in the field is complex and may be influenced by several factors in addition to levels in food and water. During a metal bioaccumulation study on catfish performed by Avenant-Oldewage and Marx (2000) in the Olifants River, the authors also state that there were no distinctive trends between localities and that variation occurred in different tissues during different seasons at the respective locations. They also stress possible effects of abiotic variables on metal availability and bioaccumulation. In this study higher salinity concentrations were recorded from the Vaal River Barrage compared to the Vaal Dam. High salinity can increase both trace metal availability (Calmano et al., 1992) and toxicity (Dwyer et al., 1992). If salinity did play a role higher metal concentrations would be expected from the Vaal River Barrage, which was not always the case. The higher water hardness recorded from the Vaal River Barrage may, on the other hand, have decreased metal toxicity to fish, and as such may have negatively influenced metal availability (Borgmann and Norwood, 1994). Sorption of heavy metals to sediment particles also reduces their bio-availability. As a result Grobler et al. (1987) believes that the turbid nature of the Vaal River System has a positive effect on water quality. Secci disc readings (Fig. 1) indicate that the Vaal Dam was more turbid during the autumn, winter and spring surveys. Only during the summer surveys were turbid conditions slightly higher at the Vaal River Barrage when compared to the Vaal Dam. If these suspended particles indeed do mitigate metal toxicity, the effect would 


\begin{tabular}{|c|c|c|c|}
\hline \multicolumn{4}{|c|}{$\begin{array}{c}\text { Table } 13 \\
\text { Table summarising instances where statistically significant } \\
\text { differences (T-test) were recorded in Clarias gariepinus } \\
\text { tissues between localities during the respective surveys }\end{array}$} \\
\hline Metal & Survey & ${ }^{1}$ Tissue & ${ }^{2}$ Locality \\
\hline \multirow{9}{*}{ Strontium } & \multirow{2}{*}{ Jan 1999} & \# Liver; \# Gill arch; * Gill filament & VD \\
\hline & & * Muscle & VRB \\
\hline & \multirow{2}{*}{ Mar 1999} & * Gill arch; * Gill filament & VD \\
\hline & & ** Liver & VRB \\
\hline & $\begin{array}{l}\text { Jun } 1999 \\
\text { Aug } 1999\end{array}$ & * Gill arch; * Gill filament & VD \\
\hline & \multirow{2}{*}{ Oct 1999} & * Gill arch; * Gill filament & VD \\
\hline & & ** Skin; $* *$ Liver & VRB \\
\hline & Feb 2000 & * Gill arch; * Gill filament & VD \\
\hline & Pooled & & \\
\hline \multirow{6}{*}{ Aluminium } & Mar 1999 & ** Muscle & VRB \\
\hline & Jun 1999 & **Liver; \#Gill arch; * Gill filament & VD \\
\hline & Aug 1999 & \# Gill arch & VRB \\
\hline & Oct 1999 & \#Gill arch & VD \\
\hline & Feb 2000 & $\begin{array}{l}* * \text { Muscle } \\
\text { \# Gill filament } \\
\end{array}$ & \begin{tabular}{|l|} 
VD \\
VRB \\
\end{tabular} \\
\hline & Pooled & \# Skin; ** Liver & VD \\
\hline \multirow{5}{*}{ Lead } & Jan 1999 & \begin{tabular}{|l} 
\# Skin \\
$* *$ Liver
\end{tabular} & \begin{tabular}{|l|} 
VD \\
VRB \\
\end{tabular} \\
\hline & Mar 1999 & \# Muscle & VRB \\
\hline & Aug 1999 & \# Muscle; * Gill Arch & VRB \\
\hline & Oct 1999 & $\begin{array}{l}\text { ** Gill arch; ** Gill filament } \\
\text { *Skin; \# Liver }\end{array}$ & \begin{tabular}{|l|} 
VD \\
VRB \\
\end{tabular} \\
\hline & Feb 2000 & \begin{tabular}{|l|} 
*Muscle \\
*Skin \\
\end{tabular} & \begin{tabular}{|l|} 
VD \\
VRB \\
\end{tabular} \\
\hline \multirow{7}{*}{ Nickel } & Jan 1999 & **Muscle & VRB \\
\hline & Mar 1999 & $\begin{array}{l}\text { **Muscle; } * * \text { Gill filament } \\
* \text { Skin }\end{array}$ & \begin{tabular}{|l|} 
VD \\
VRB
\end{tabular} \\
\hline & Jun 1999 & $\begin{array}{l}\text { \#Skin; **Muscle } \\
\text { *Gill filament }\end{array}$ & \begin{tabular}{|l|} 
VD \\
VRB \\
\end{tabular} \\
\hline & Aug 1999 & $\begin{array}{l}\text { *Gill arch; * Gill filament } \\
\text { *Liver; *muscle }\end{array}$ & \begin{tabular}{|l|} 
VD \\
VRB \\
\end{tabular} \\
\hline & Oct 1999 & $\begin{array}{l}\text { *Gill arch; \#Gill filament } \\
\text { \#Skin; * Muscle }\end{array}$ & \begin{tabular}{|l|} 
VD \\
VRB \\
\end{tabular} \\
\hline & Feb 2000 & $\begin{array}{l}\text { *Gill arch } \\
\text { \#Skin } \\
\end{array}$ & \begin{tabular}{|l|l|} 
VD \\
VRB \\
\end{tabular} \\
\hline & Pooled & $\begin{array}{l}\text { *Gill arch; *Gill filament } \\
\text { *Skin }\end{array}$ & \begin{tabular}{|l|} 
VD \\
VRB \\
\end{tabular} \\
\hline \multicolumn{4}{|c|}{$\begin{array}{l}1=\text { Tissues for which significant differences in metal concentration between } \\
\text { localities were recorded. } \\
2=\text { Locality for which the highest metal concentration was recorded. } \\
V D=\text { Vaal Dam VRB } \quad \text { Vaal River Barrage } \\
\begin{array}{ll}* *=p<0.050 \quad \#=p<0.010 \quad *=p<0.001\end{array}\end{array}$} \\
\hline
\end{tabular}

effect (metal concentrations 'diluted' during periods of flooding) must also be kept in mind. Depending on the severity of the flood, the dilution effect will be greater than the re-suspension of metals from sediment resulting from the flood which would normally increase metal concentrations (e.g. Brown, 1977). The effect of the floods is thus expected to have a greater influence on the Vaal River Barrage than the Vaal Dam. Despite the severity of the February 2000 flood (Figure 1), the dilution effect was not pronounced at either the Vaal Dam or Vaal River Barrage. Effects resulting from increased flow rate as a result of the floods are thus unlikely to have contributed to the fact that higher metal concentrations were not recorded from the Vaal River Barrage.

Clements and Rees (1997) discussed other possible explanations that may also shed more light on differences in tissue metal concentrations between localities. They stated that if fish growth or age is not determined, differences in growth rates or age between sites might contribute to differences in metal levels. In the present study fish were collected using gill nets. As a result fish of a specific size were collected. However, growth rate and age may still be important even if fish within the same size-class are selected for metal analysis (Clements and Rees, 1997). This is a possible shortcoming in the current study. In future, age determinations may have to be performed, after which fish will need to be divided into age-classes for metal analysis. The authors further state that potential negative effects of heavy metals may be offset by higher temperatures and increased prey availability. Slightly higher temperatures were recorded in the Vaal River Barrage during the summer, spring and autumn months (Fig. 1). Their study was however concerned with brown trout. It is unlikely that the slight differences in temperature observed in the current study will have any significantly positive effect on fish in the Vaal River Barrage, compared to fish in the Vaal Dam. The higher number of piscivorous birds at the Vaal River Barrage when compared to the Vaal Dam (personal observation) at the time of conduct of the study, may also indicate higher prey availability. This is however unlikely to be the case - a bird sanctuary is located near the Vaal River Barrage which could explain the higher numbers of birds observed. Furthermore, largemouth bass

thus be greater at the Vaal Dam. Under aquaculture conditions flushing rates have been shown to influence metal accumulation in trout (Davidson et al., 2009) and sea bass (Deviller et al., 2005). Furthermore, flow rates can influence the results obtained when analysing water samples collected from rivers for nickel. Snodgrass (1980) states that particulate matter is flow sensitive and will depend on the amount of rainfall in the river catchment. As a result he believes such samples do not provide a true picture of the total distribution and transport of nickel. The variability in flow rate between surveys (Fig. 1) in the Vaal River Barrage thus may have masked any trends. Increased flow rate results from heavy rain. High rainfall often leads to a lowering of $\mathrm{pH}$, which promotes solubility of heavy metal compounds (Hahne and Kroontje, 1973). The dilution
(Micropterus salmoides) were collected from the Vaal River Barrage, but not the Vaal Dam during the 1999/2000 surveys. These fish are aggressive predators (Skelton, 1993). The fact that food overlap exists between C. gariepinus and other alien predator species (Dörgeloh, 1996; Dörgeloh, 1994; Skelton, 1993), combined with the large amount of piscivorous birds leads to the assumption that prey numbers may well be lower in the Vaal River Barrage as a result of predation pressure. Apart from these 2 factors, Giesy and Wiener (1977) stated that 'limitations of sample sizes and sampling procedures usually make it difficult to determine the degree to which age, location, time of collection, and other factors affect variance in concentrations'. Furthermore, largemouth bass have since been collected from the Vaal Dam locality during recent (2009/2010) parasite 
Table 14

Table depicting the ranking of metal concentrations in $\mathbf{5}$ different tissue types, collected from the Vaal Dam and Vaal River Barrage during 7 surveys conducted between November 1998 and February 2000.

\begin{tabular}{|c|c|c|c|c|c|c|c|c|c|}
\hline \multicolumn{10}{|l|}{ Vaal Dam } \\
\hline Metal & \multicolumn{9}{|c|}{ Ranking of accumulation from highest to lowest concentration } \\
\hline \multirow{4}{*}{\begin{tabular}{|l} 
Strontium \\
Aluminium \\
Lead \\
Nickel \\
\end{tabular}} & Gill arch & $>$ & Gill filament & $>$ & Skin & $>$ & Liver & $>$ & Muscle \\
\hline & Gill filament & $>$ & Skin & $>$ & Liver & $>$ & Gill arch & $>$ & Muscle \\
\hline & Gill arch & $>$ & Gill filament & $>$ & Liver & $>$ & Muscle & $>$ & Skin \\
\hline & Gill arch & $>$ & Gill filament & $>$ & Muscle & $>$ & Liver & $>$ & Skin \\
\hline \multicolumn{10}{|c|}{ Vaal River Barrage } \\
\hline Metal & \multicolumn{9}{|c|}{ Ranking of accumulation from highest to lowest concentration } \\
\hline \multirow{4}{*}{$\begin{array}{l}\text { Strontium } \\
\text { Aluminium } \\
\text { Lead } \\
\text { Nickel }\end{array}$} & Gill arch & $>$ & Gill filament & $>$ & Muscle & $>$ & Liver & $>$ & Skin \\
\hline & Gill filament & $>$ & Skin & $>$ & Gill arch & $>$ & Liver & $>$ & Muscle \\
\hline & Gill arch & $>$ & Gill filament & $>$ & Muscle & $>$ & Liver & $>$ & Skin \\
\hline & Gill arch & $>$ & Skin & $>$ & Muscle & $>$ & Gill filament & $>$ & Liver \\
\hline
\end{tabular}

collection surveys (observation by author). Another host-related aspect that could be investigated in future studies is genderspecific interactions (e.g. Pyle et al., 2005). Pollutant concentrations and biological responses in fish relate to cyclic physiological changes that are linked not only to food availability, but also reproduction (Nesto et al., 2007; Marijić and Raspor, 2010). Highest metal concentrations are indeed often found in gonads (e.g. Alquezar et al., 2006). In the current study, tissue concentrations in gonads were however not examined.

Köck et al. (1996) stated that metal uptake may be enhanced during summer as a result of increasing metabolic rates. Most significant differences recorded between surveys were concerned with metal concentrations in muscle and liver tissues. This was however not always the case, as exemplified by the Al concentrations recorded. Possible confounding factors could be any of the variables listed in Fig. 1 or even changes in water or sediment concentrations. It is also possible that decreased metabolism during winter (resulting from low temperatures) may have played a role.

With regard to accumulation of the 4 metals in question in fish tissue, the general order (ranked in order of decreasing concentrations) was found to be: gill arch $>$ gill filament $>$ muscle $>$ liver $>$ skin. Very high standard deviation values were however recorded, indicating that the ranking obtained should be interpreted with caution. Kargin (1998) recorded highest metal concentrations (including $\mathrm{Pb}$ ) in liver and gills, and lowest concentrations in muscle, of Capoeta barroisi from Turkey. Kargin (1998), after reviewing accumulation patterns of metals, further stated that this result is expected. The liver is highly active in the uptake and storage of pollutants and other non-nutritive molecules (Hopson and Wessels, 1990; Sorensen, 1991; Kimball, 1983), while active and passive exchanges occur between the animal and the aquatic environment through the gills (Eckert et al., 1988; Kargin, 1998). Afonso et al. (2007) found highest mercury concentrations in liver (followed by muscle and skin respectively), while Dang and Wang (2009) also found cadmium concentrations to be highest in this organ. Y1lmaz et al. (2007) recorded highest $\mathrm{Pb}$ concentrations in gill and liver tissue, as did Al-Kahtani (2009), Su et al. (2009) and Pereira et al. (2010a) with reference to liver tissue. Visnjic-Jeftic et al. (2010) confirmed the general trend (i.e. high concentration in gills) for $\mathrm{Al}$ and $\mathrm{Sr}$ and found lowest concentrations in muscle. Other authors that found high metal concentrations in liver and/or gill tissue include Deviller et al. (2005),
Yang et al. (2007), Vinodhini and Narayanan (2008) and Heier et al. (2009). Robinson and Avenant-Oldewage (1997) also speculated that the large surface area of the gills, and the large amount of water that passes over them, further facilitate metal uptake. High metal concentrations were also recorded from gill arches. Similarly Seymore et al. (1995) found high metal concentrations in vertebrae, and recommended the use of bony tissue for the analysis of $\mathrm{Pb}$ and $\mathrm{Sr}$ in fish. Playle (1998) concludes that metal-gill modelling is indeed a good framework for understanding and predicting metal toxicity to fish. The relevance of this statement was confirmed by Pereira et al. (2010b) who demonstrated that gills do reflect water contamination.

While findings in thus study do appear to concur with the general trend discussed above for the majority of metals (i.e. highest concentration in liver and gill tissues and lowest concentrations in muscle and skin tissues), exceptions have been recorded. Y1lmaz et al. (2010) found higher Ni concentrations in skin compared to liver. Reynders et al. (2008) found highest $\mathrm{Cd}$ concentrations in the intestine and kidney, followed by gills, liver and finally muscle. Rauf et al. (2009) in turn found highest Cd concentrations in liver and lowest concentrations in gill tissue after examining gills, kidney, liver, skin, muscle and scales. De Boeck et al (2010) found only Cu concentrations to be highest in liver. The remaining metals (including $\mathrm{Ni}$ and $\mathrm{Pb}$ ) were highest in either the kidney or rectal gland. Dural et al. (2007) found highest metal concentrations (including $\mathrm{Pb}$ ) in muscle tissue after examining muscle, gill, liver and gonad tissues. These apparent discrepancies in available literature are due to variability between fish species, tissues and metals examined (e.g. Mendil et al., 2005; Kraemer et al., 2005; Arain et al., 2008; Uysal et al., 2008; Lakshmanan et al., 2009; Mathews and Fisher, 2009; Mendil et al., 2010). Schmitt et al. (2007) as well as Qiao-qiao et al. (2007) reported within-site variability with regards to metal concentrations in different tissue types. Some fish species also appear to possess an adaptation capability to heavy metal loads (Shah and Altindağ, 2005). The observed variability is however not only restricted to fish species and their tissue. Mohan et al. (2007) found no regular pattern of metal deposition at different trophic levels. Be that as it may, it seems that highest metal concentrations are consistently found in the liver and gills of C. gariepinus (e.g. Gbem et al., 2001), as is also reflected in the current study.

A study by Retief et al. (2009) in the Vaal Dam also found no seasonal trend with regards to metal bioaccumulation in 
host (largemouth yellowfish) tissue. They did however identify a seasonal trend with regards to bioaccumulation in the tapeworm parasite (Bothriocephalus acheilognathi). Furthermore, higher mean metal concentration values were recorded from parasite tissue compared to host tissue. Woelfl et al. (2008) also found that Diphyllobothrium latum bioconcentrate more trace elements than their host (Oncorhynchus mykiss). Furthermore, $\mathrm{Pb}$ was found in the parasite but not in any fish organs, indicating that endoparasitic flatworms as sensitive bio-indicators may have an 'early warning' biological monitoring function. OyooOkoth et al. (2010) however caution that partitioning of metals in the parasite/host assemblage may occur. This statement implies a need for baseline data on metal accumulation in both host and parasite species to determine the degree of partitioning. Further studies on other fish species and parasites might not only be useful to confirm trends, but may have interesting implications with regards to biological monitoring practices in the Vaal River system.

\section{Conclusions}

Heavy metals did accumulate in C. gariepinus tissues. While $\mathrm{Sr}$ concentrations in gills were more often significantly $(p<0.05)$ higher in fish from the Vaal Dam, no further clear trends emerged with regards to differences between localities (Vaal Dam and Vaal River Barrage) or surveys. Highest metal concentrations were recorded in gill (both filaments and arches), followed by muscle, liver and lastly skin. This general trend appears to be in agreement with trends observed by other workers and reported in the literature. Comparison of tissue metal concentrations recorded in the current study with that recorded for other species in the literature once again strengthened the notion of interspecies bioaccumulation differences. Differences in tissue metal concentrations in C. gariepinus between the current study and results from available literature also accentuated the importance of factors that influence the concentrations and bioavailability of trace metals.

\section{Acknowledgements}

The authors would like to thank the former Rand Afrikaans University (now University of Johannesburg) for financial and logistical support and the National Research Foundation for financial assistance.

\section{References}

AFONSO C, LOURENÇO HM, DIAS A, NUNES ML and CASTRO M (2007) Contaminant metals in Black scabbard fish (Aphanopus carbo) caught off Madeira and the Azores. Food Chem. 101 120-125.

ALI MHH and FISHAR MRA (2005) Accumulation of trace metals in some benthic invertebrate and fish species relevant to their concentration in water and sediment of lake Qarun, Egypt. Egypt. J. Aquat. Res. 31 (1) 289-301.

AL-KAHTANI MA (2009) Accumulation of heavy metals in Tilapia fish (Oreochromis niloticus) from Al-Khadoud Spring, Al-Hassa, Saudi Arabia. Am. J. Appl. Sci. 6 2024-2029.

ALLERT AL, FAIRCHILD JF, SCHMITT CJ, BESSER JM, BRUMBAUGH WG and OLSON SJ (2009) Effects of mining-derived metals in riffle-dwelling benthic fishes in Southeast Missouri, USA. Ecotoxicol. Environ. Safety 72 1642-1651.

ALQUEZAR R, MARKICH SJ and BOOTH DJ (2006) Metal accumulation in the smooth toadfish, Tetractenos glaber, in estuaries around Sydney, Australia. Environ. Pollut. 142 123-131.

ALQUEZAR R, MARKICH SJ and TWINING JR (2008) Comparative accumulation of ${ }^{109} \mathrm{Cd}$ and ${ }^{75} \mathrm{Se}$ from water and food by an estuarine fish (Tetractenos glaber). J. Environ. Radioactiv. 99 167-180.

ANNUNE PA, EBELE SO and OLADIMEJI AA (1994) Acute toxicity of cadmium to juveniles of Clarias gariepinus (Teugels) and Oreochromis niloticus (Trewavas). J. Environ. Sci. Health A 29 (7) 1357-1365.

ARAIN MB, KAZI TG, JAMALI MK, JALBANI N, AFRIDI HI and SHAH A (2008) Total dissolved and bioavailable elements in water and sediments samples and their accumulation in Oreochromis mossambicus of polluted Manchar Lake. Chemosphere 70 1845-1856.

AVENANT-OLDEWAGE A and MARX HM (2000) Bioaccumulation of chromium, copper and iron in the organs and tissues of Clarias gariepinus in the Olifants River, Kruger National Park. Water SA 26 (4) 569-582.

BARNHOORN I, VAN VUREN JHJ and DU PREEZ HH (1999) Sublethal effects of manganese on the carbohydrate metabolism of Oreochromis mossambicus after acute and chronic exposure. S. Afr. J. Zool. 34 (3) 102-107.

BAKER JP and SCHOFIELD CL (1982) Aluminium toxicity to fish in acidic waters. Water Air Soil Pollut. 18 289-309.

BENIJTS-CLAUS C and BENIJTS F (1975) The effects of low lead and zinc concentrations on the larval development of the mud-crab Rhithropanopeus harrisii Gould. In: Koeman JH and Strik JJTWA (eds.) Sublethal Effects of Toxic Chemicals on Aquatic Animals. Elsevier Scientific Publishing Company, Amsterdam. 43-52.

BENOIT DA (1975) Chronic effects of copper on survival, growth, and reproduction of the bluegill (Lepomis macrochirus). Trans. Am. Fish. Soc. 2 353-358.

BERVOETS L, KNAEPKENS G, EENS M and BLUST R (2005) Fish community responses to metal pollution. Environ. Pollut. 138 338-349.

BIRGE WJ and BLACK JA (1980) Aquatic toxicology of nickel. Ch. 13. In: Nriagu JO (ed.) Nickel in the Environment. John Wiley and Sons, New York.

BIRUNGI Z, MASOLA B, ZARANYIKA MF, NAIGAGA I and MARSHALL B (2007) Active biomonitoring of trace heavy metals using fish (Oreochromis niloticus) as bioindicators species. The case of Nakivubo wetland along Lake Victoria. Phys. Chem. Earth 32 1350-1358.

BLIGNAUT J and VAN HEERDEN J (2009) The impact of water scarcity on economic development initiatives. Water SA 35 (4) 415-420.

BORGMANN U and NORWOOD WP (1994) Kinetics of excess (above background) copper and zinc in Hyalella azteca and their relationship to chronic toxicity. Can. J. Fish. Aquat. Sci. 52 864-874.

BRAUNE E and ROGERS KH (1987) The Vaal River catchment: problems and research needs. Foundation for Research Development, Pretoria.

BROWN BE (1977) Effects of mine drainage on the River Hayle, Cornwall: A) Factors affecting concentrations of copper, zinc and iron in water, sediments and dominant invertebrate fauna. Hydrobiol. 52 (2-3) 221-233.

BRYAN GW (1976) Some aspects of heavy metal tolerance in aquatic organisms. In: Lockwood APM (ed.) Effects of pollutants on aquatic organisms. Cambridge University Press, Cambridge.

BUERGEL PM and SOLTERO RA (1983) The distribution and accumulation of aluminium in rainbow trout following a whole-lake alum treatment. J. Freshwater Ecol. 2 (1) 37-44.

BURTON DT, MORGAN EL and CAIRNS J (1972) Mortality curves of bluegills (Lepomis macrochirus Rafinesque) simultaneously exposed to temperature and zinc stress. Trans. Am. Fish. Soc. 3 435-442.

CALMANO W, AHLF W and BENING JC (1992) Chemical mobility and bioavailability of sediment-bound heavy metals influenced by salinity. Hydrobiol. 235/236 605-610.

CARR HP, CARINO FA, YANG MS and WONG MH (1998) Characterization of the cadmium-binding capacity of Chlorella vulgaris. Bull. Environ. Contam. Toxicol. 60 433-440.

CASTRO-GONZÁLEZ MI and MÉNDEZ-ARMENTA M (2008) Heavy metals: Implications associated to fish consumption. Ecotoxicol. Environ. Safety 26 263-271. 
CHAPMAN PM, WANG F, JANSSEN C, PERSOONE G and ALLEN, HE (1998) Ecotoxicology of metals in aquatic sediments: binding and release, bioavailability, risk assessment, and remediation. Can J. Fish. Aquat. Sci. 55 2221-2243.

CLEMENTS, WH and REES DE (1997) Effects of heavy metals on prey abundance, feeding habits, and metal uptake of brown trout in the Arkansas River, Colorado. Trans. Am. Fish. Soc. 126 774-785.

COETZEE PP (1993) Determination and speciation of heavy metals in sediments of the Hartbeespoort Dam by sequential chemical extraction. Water SA 19 (4) 291-300.

CRAFFORD D and AVENANT-OLDEWAGE A (2009) Application of a fish health assessment index and associated parasite index to Clarias gariepinus (Teleostei: Clariidae) in the Vaal River system, South Africa. Afr. J. Aquat. Sci. 34 (3) 261-272.

DANG F and WANG W-X (2009) Assessment of tissue-specific accumulation and effects of cadmium in a marine fish fed contaminated commercially produced diet. Aquat. Toxicol. 95 248-255.

DAVIDSON J, GOOD C, WELSH C, BRAZIL B and SUMMERFELT S (2009) Heavy metal and waste metabolite accumulation and their potential effect on rainbow trout performance in a replicated water reuse system operated at low or high system flushing rates. Aquacult. Eng. 41 136-145.

DE BOECK G, EYCKMANS M, LARDON I, ROBBAERS R, SINHA AK and BLUST R (2010) Metal accumulation and metallothionein induction in the spotted dogfish Scyliorhinus canicula. Comp. Biochem. Physiol. A 155 503-508.

DEVILLER G, PALLUEL O, ALIAUME C, ASANTHI H, SANCHEZ W, MA FRANCO NAVA, BLANCHETON J-P and CASELLAS $\mathrm{C}$ (2005) Impact assessment of various rearing systems on fish health using multibiomarker response and metal accumulation. Ecotoxicol. Environ. Safety 61 89-97.

DICKSON W (1983) Liming toxicity of aluminium to fish. Vatten 39 400-404.

DÖRGELOH WG (1994) Diet and food selection of Barbus aeneus, Clarias gariepinus and Oncorhynchus mykiss in a clear man-made lake, South Africa. Water SA 20 (1) 91-98.

DÖRGELOH WG (1996) Food overlap between the alien Oncorhynchus mykiss and the indigenous fish species Barbus aeneus and Clarias gariepinus in a man-made lake, South Africa. Water SA 22 (1) 79-83.

DURAL M, GÖKSU MZL and ÖZAK AA (2007) Investigation of heavy metal levels in economically important fish species captured from the Tuzla lagoon. Food Chem. 102 415-421.

DWYER FJ, BURCH SA, INGERSOLL CG. and HUNN JB (1992) Toxicity of trace element and salinity mixtures to striped bass (Morone saxatilis) and Daphnia magna. Environ. Toxicol. Chem. 11 513-520.

ECKERT R, RANDALL D and AUGUSTINE G (1988) Animal Physiology; Mechanisms and Adaptations. WH Freeman and Company, New York.

ENSERINK EL, MAAS-DIEPEVEEN JL and VAN LEEUWEN CJ (1991) Combined effects of metals; an ecotoxicological evaluation. Water Res. 25 (6) 679-687.

GALVIN RM (1996) Occurrence of metals in waters: an overview. Water SA 22 (1) 7-18.

GBEM TT, BALOGUN JK, LAWAL FA and ANNUNE PA (2001) Trace metal accumulation in Clarias gariepinus (Teugels) exposed to sublethal levels of tannery effluent. Sci. Total. Environ. 271 1-9.

GHATAK DB, HOSSAIN M and KONAR SK (1987) Acute toxicity of mixture of heavy metal cadmium and pesticide phosphamidon to plankton, worm and fish. Environ. Ecol. 5 (4) 751-755.

GIESY JP and WIENER JG (1977) Frequency distributions of trace metal concentrations in five freshwater fishes. Trans. Am. Fish. Soc. 106 (4) 393-402.

GOLDSTEIN RM, BRIGHAM ME and STAUFFER C (1995) Comparison of mercury concentrations in liver, muscle, whole bodies, and composites of fish from the Red River of the North. Can. J. Fish. Aquat. Sci. 53: 244-252.

GOUWS K and COETZEE PP (1997) Determination and partitioning of heavy metals in sediments of the Vaal Dam System by sequential extraction. Water SA 23 (3) 217-226.
GROBLER DC, TOERIEN DF and ROSSOUW JN (1987) A review of sediment/water quality interaction with particular reference to the Vaal River system. Water SA 13 (1) 15-22.

GUTLEB AC, KRANZ A, NECHAY G and TOMAN A (1998) Heavy metal concentrations in livers and kidneys of the otter (Lutra lutra) from central Europe. Bull. Environ. Contam. Toxicol. 60 273-279.

HAHNE HCH. and KROONTJE W (1973) Significance of pH and chloride concentration on behavior of heavy metal pollutants: mercury (II), cadmium (II), zinc (II), and lead (II). J. Environ. Quality 2 (4) 444-448.

HALL BD, ROSENBERG DM and WIENS AP (1998) Methyl mercury in aquatic insects from an experimental reservoir. Can. J. Fish. Aquat. Sci. 55 2036-2047.

HARTUNG R (1973) Biological effects of heavy metal pollutants in water. In: SK Dhar SK (ed.) Metal Ions in Biological Systems; Studies of Some Biochemical and Environmental Problems. Plenum Press, New York. 161-172.

HAUX C, LARSSON A, LITHNER G and SJÖBECK M (1986) A field study of physiological effects on fish in lead-contaminated lakes. Environ. Toxicol. Chem. 5 283-288.

HEIER LS, LIEN IB, STRØMSENG AE, LJØNES M, ROSSELAND BO, TOLLEFSEN K-E and SALBU B (2009) Speciation of lead, copper, zinc and antimony in water draining a shooting rangeTime dependant metal accumulation and biomarker responses in brown trout (Salmo trutta L.) Sci. Total. Environ. 407 4047-4055.

HEM JD (1977) Reactions of metal ions at surfaces of hydrous iron oxide. Geochim. Cosmochim. Acta 41 527-538.

HODSON PV (1988) The effect of metal metabolism on uptake, disposition and toxicity in fish. Aquat. Toxicol. 11 3-18.

HOGSTRAND C and HAUX C (1991) Binding and detoxification of heavy metals in lower vertebrates with reference to metallothionein Comp. Biochem. Physiol. 100C 137-141.

HOPSON JL and WESSELS NK (1990) Essentials of Biology. McGraw Hill Publishing Company, New York.

HOUBA C, REMACLE J, DUBOIS D. and THOREZ J (1983) Factors affecting the concentrations of cadmium, zinc, copper and lead in the sediments of the Verde River. Water Res. 17 1281-1286.

JACKSON VA, PAULSE AN, VAN STORMBROEK T and KHAN W (2007) Investigation into metal contamination of the Berg River, Western Cape, South Africa. Water SA 33 (2) 175-182.

JACKSON VA, PAULSE AN, ODENDAAL JP and KHAN W (2009) Investigation into the metal contamination of the Plankenburg and Diep Rivers, Western Cape, South Africa. Water SA 35 (3) 289-300.

JAMES R, SAMPATH K and SELVAMANI P (1998) Effect of EDTA on reduction of copper toxicity in Oreochromis mossambicus (Peters). Bull. Environ. Contam. Toxicol. 60 487-493.

JOHNSON CA (1986) The regulation of trace element concentrations in river and estuarine waters contaminated with acid mine drainage: The adsorption of $\mathrm{Cu}$ and $\mathrm{Zn}$ on amorphous $\mathrm{Fe}$ oxyhydroxides. Geochim. Cosmochim. Acta 50 2433-2438.

KARGIN F (1998) Metal concentrations in tissues of the freshwater fish Capoeta barroisi from the Seyhan River (Turkey). Bull. Environ. Contam. Toxicol. 60 822-828.

KIDWELL JM, PHILLIPS LJ and BIRCHARD GF (1995) Comparative analysis of contaminant levels in bottom feeding and predatory fish using the national contaminant biomonitoring program data. Bull. Environ. Contam. Toxicol. 54 919-923.

KIMBALL JW (1983) Biology. Addison-Wesley Publishing Company, Massachusetts.

KLAASEN CD (1976) Biliary excretion of metals. Drug. Metab. Rev. 5 (2) 165-196.

KLIGOUR BW (1991) Cadmium uptake from cadmium-spiked sediments by four freshwater invertebrates. Bull. Environ. Contam. Toxicol. 47 70-75.

KÖCK G, HOFER R and WÖGRATH S (1995) Accumulation of trace metals (Cd, $\mathrm{Pb}, \mathrm{Cu}, \mathrm{Zn}$ ) in Arctic char (Salvelinus alpinus) from oligotrophic Alpine lakes: relation to alkalinity. Can. J. Fish. Aquat. Sci. 52 2367-2376.

KÖCK G, TRIENDL M. and HOFER R (1996) Seasonal patterns of metal accumulation in Arctic char (Salvelinus alpinus) from an oligotrophic Alpine lake related to temperature. Can. J. Fish. Aquat. Sci. 53 780-786. 
KRAEMER LD, CAMPBELL PGC and HARE L (2005) Dynamics of $\mathrm{Cd}, \mathrm{Cu}$ and $\mathrm{Zn}$ accumulation in organs and sub-cellar fractions in field transplanted juvenile yellow perch (Perca flavescens). Environ. Pollut. 138 324-337.

KWONG RWM and NIYOGI S (2009) The interactions of iron with other divalent metals in the intestinal tract of a freshwater teleost, rainbow trout (Oncorhynchus mykiss). Comp. Biochem. Physiol. C 150 442-449.

LAKSHMANAN R, KESAVAN K, VIJAYANAND P, RAJARAM V and RAJAGOPAL S (2009) Heavy metals accumulation in five commercially important fishes of Parangipettai, Southeast Coast of India. Adv. J. Food Sci. Technol. 1 (1) 63-65.

LANGE TR, ROYALS HE and CONNOR LL (1993) Influence of water chemistry on mercury concentration in largemouth bass from Florida lakes. Trans. Am. Fish. Soc. 122 74-84.

LITERATHY P and LASZLO F (1977) Uptake and release of heavy metals in the bottom silt of recipients. In: Golterman HL (ed.) Interactions between Sediments and Fresh Water. Dr W Junk B.V. Publishers, The Hague.

LLOYD R (1992) Pollution and Freshwater Fish. Fishing News Books, London.

LOGANATHAN P and BURAU RG (1973) Sorption of heavy metal ions by a hydrous manganese oxide. Geochim. Cosmochim. Acta. 37 1277-1293.

LOWE TP, MAY TW, BRUMBAUGH WG and KANE DA (1985) National contaminant biomonitoring program: concentrations of seven elements in freshwater fish, 1978-1981. Arch. Environ. Contam. Toxicol. 14 363-388.

MAAGE A (1990) Comparison of cadmium concentrations in Atlantic salmon (Salmo salar) fry fed different commercial feeds. Bull. Environ. Contam. Toxicol. 44 770-775.

MANCE G (1987). Pollution Threat of Heavy Metals in Aquatic Environments. Elsevier Applied Science, London.

MARIJIĆ VF and RASPOR B (2010) The impact of fish spawning on metal and protein levels in gastrointestinal cytosol of indigenous European chub. Comp. Biochem. Physiol. C 152 133-138.

MARX HM (1996) Evaluation of a Health Assessment Index with reference to metal bioaccumulation in Clarias gariepinus and aspects of the biology of the parasite Lamproglena clariae. M.Sc. Dissertation, Rand Afrikaans University, Johannesburg.

MATHEWS T and FISHER NS (2009) Dominance of dietary intake of metals in marine elasmobranch and teleost fish. Sci. Total Environ. 407 5156-5161.

MENDIL D, ULUÖZLÜ ÖD, HASDEMIR E, TÜZEN M, SARI H and SUIÇMEZ M (2005) Determination of trace metal levels in seven fish species in lakes in Tokat, Turkey. Food Chem. 90 175-179.

MENDIL R, ÜNAL ÖF, TÜZEN M and SOYLAK M (2010) Determination of trace metals in different fish species and sediments from the River Yeşilırmak in Tokat, Turkey. Food Chem. Toxicol. 48 1383-1392.

MERLINI M and POZZI G (1977) Lead and freshwater fishes: Part 1 lead accumulation and water pH. Environ. Pollut. 12 167-172.

MOHAN D, CHAUDHARY A and GAUR S (2008) Patterns of trace metals accumulation in different Trophic Levels of Lake Kailana, Jodhpur (India). Proc. Taal2007: The 12 ${ }^{\text {th }}$ World Lake Conference. 373-377.

MOODLEY KG, BAIJNATH H, SOUTHWAY-AJULU FA, MAHARAJ $\mathrm{S}$ and CHETTY SR (2007) Determination of $\mathrm{Cr}, \mathrm{Pb}$ and $\mathrm{Ni}$ in water, sludge and plants from settling ponds of a sewage treatment works. Water SA 33 (5) 723-728.

MOORE JW and RAMAMOORTHY S (1984) Heavy Metals in Natural Waters: Applied Monitoring and Impact Assessment. Springer-Verlag, New York.

MURAMOTO S (1980) Effect of complexans (EDTA, NTA and DTPA) on the exposure to high concentrations of cadmium, copper, zinc and lead. Bull. Environ. Contam. Toxicol. 25 941-946.

NESTO N, ROMANO S, MOSCHINO V, MAURI M and DA ROS L (2007) Bioacumulation and biomarker responses of trace metals and micro-organic pollutants in mussels and fish from the Lagoon of Venice, Italy. Marine Poll. Bull. 55 469-484.

NEUMAN ISA, VAN ROSSUM C, BRETSCHNEIDER F, TEUNIS PFM and PETERS RC (1991) Biomonitoring: cadmium deteriorates electro-orientation performance in catfish. Comp. Biochem. Physiol. 100C 259-262.

NORRGREN L, GLYNN AW and MALMBORG O (1991) Accumulation and effects of aluminium in the minnow (Phoxinus phoxinus L.) at different pH levels. J. Fish Biol. 39 833-847.

OTERO-MURAS I, FRANCO-URíA A, ALONSO AA and BALSACANTO E (2010) Dynamic multi-compartmental modelling of metal bioaccumulation in fish: Identifiability implications. Environ. Model. Softw. 25 344-353.

OYOO-OKOTH E, ADMIRAAL W, OSANO O, HOITINGA L and KRAAK MHS (2010) Metal specific partitioning in a parasite-host assemblage of the cestode Ligula intestinalis and the cyprinid fish Rastrineobola argentea. Sci. Total Environ. 408 1557-1562.

PALANIAPPAN PL RM and KARTHIKEYAN S (2009)

Bioaccumulation and depuration of chromium in the selected organs and whole body tissues of freshwater fish Cirrhinus Mrigala individually and in binary solutions with nickel. J. Environ. Sci. 21 229-236.

PANDEY S, PARVEZ S, ANSARI RA, ALI M, KAUR M, HAYAT F, AHMAD F and RAISUDDIN S (2008) Effects of exposure to multiple trace metals in biochemical histological and ultrastructural features of gills of a freshwater fish, Channa punctata Bloch. Chem. Biol. Interact. 174 183-192.

PEREIRA P, DE PABLO H, PACHECO M and VALE C (2010a) The relevance of temporal and organ specific factors in metals accumulation and biochemical effects in feral fish (Liza aurata) under a moderate contamination scenario. Ecotoxicol. Environ. Safety 73 805-816.

PEREIRA P, DE PABLO H, VALE C and PACHECO M (2010b) Combined use of environmental data and biomarkers in fish (Liza aurata) inhabiting a eutrophic and metal-contaminated coastal system - gills reflect environmental contamination. Mar. Environ. Res. 69 53-62.

PHUONG PK, SON CPN, SAUVAIN JJ and TARRADELLAS J (1998) Contamination by PCB's, DDT's, and heavy metals in sediments of Ho Chi Minh city's canals, Viet Nam. Bull. Environ. Contam. Toxicol. 60 347-354.

PICKERING QH (1974) Chronic toxicity of nickel to the fathead minnow. J. WPCF 46 (4) 660-765.

PLAYLE RC (1998) Modelling metal interactions at fish gills. Sci. Total Environ. 219 147-163.

PRASATH PMD AND KHAN H (2008) Impact of Tsunami on the heavy metal accumulation in water, sediments and fish at Poompuhar coast, Southeast Coast of India. E-Journal Chem. 5 (1) 16-22.

PURVES D (1985) Trace-element Contamination of the Environment. Elsevier, Amsterdam.

PYLE GG, RAJOTTE JW and COUTURE P (2005) Effects of industrial metals on wild fish populations along a metal contamination gradient. Ecotoxicol. Environ. Safety 61 287-312.

QIAO-QIAO C, GUANG-WEI Z and LANGDON A (2007) Bioaccumulation of heavy metals in fishes from Taihu Lake, China. J. Environ. Sci. 19 1500-1504.

RADTKE RL (1989) Strontium-calcium concentration ratios in fish otoliths as environmental indicators. Comp. Biochem. Physiol. 92A (2) 189-193.

RAUF A, JAVED M and UBAIDULLAH M (2009) Heavy metal levels in three major carps (Catla catla, Labeo Rohita and Cirrhina Mrigala) from the river Ravi, Pakistan. Pakistan Vet. J. 29 (1) 24-26.

RETIEF N-R, AVENANT-OLDEWAGE A and DU PREEZ HH (2009) Seasonal study on Bothriocephalus as indicator of metal pollution in yellowfish, South Africa. Water SA 35 (3) 315-322.

REYNDERS H, BERVOETS L, GELDERS M, DE COEN WM and BLUST R (2008) Accumulation and effects of metals in caged carp and resident roach along a metal pollution gradient. Sci. Total Environ. 391 82-95.

ROBINSON J and AVENANT-OLDEWAGE A (1997) Chromium, copper, iron and manganese bioaccumulation in some organs and tissues of Oreochromis mossambicus from the lower Olifants River, inside the Kruger National Park. Water SA 23 (4) 387-403. 
ROEBUCK BD (1992) Heavy metals. In: Smith RP (ed.) .A Primer of Environmental Toxicology Lea and Febiger, Philadelphia.

ROSALES-HOZ L and CARRANZA-EDWARDS A (1998) Heavy metals in sediments from Coatzacoalcos River, Mexico. Bull. Environ. Contam. Toxicol. 60 553-561.

SCHMITT CJ, BRUMBAUGH WG and MAY TW (2007) Accumulation of metals in fish from lead-zinc mining areas of southeastern Missouri, USA. Ecotoxicol. Environ. Safety 67 14-30.

SCHRODER SL, KNUDSEN CM and VOLK EC (1995) Marking salmon fry with strontium chloride solutions. Can. J. Fish. Aquat. Sci. 52 1141-1149.

SEYMORE T, DU PREEZ HH and VAN VUREN JHJ (1995) Manganese, lead and strontium bioaccumulation in the tissues of the yellowfish, Barbus marequensis from the lower Olifants River, Eastern Transvaal. Water SA 21 (2) 159-172.

SHINN C, DAUBA F, GRENOUILLET G, GUENARD G and LEK S (2009) Temporal variation of heavy metal contamination in fish of the river lot in southern France. Ecotoxicol. Environ. Safety 72 1957-1965.

SHAH SL and ALTINDAĞ A (2005) Effects of Heavy Metal Accumulation on the 96-h $\mathrm{LC}_{50}$ Values in Tench Tinca tinca L., 1758. Turk. J. Vet. Anim. Sci. 29 139-144.

SHRIADAH MA (1998) Metals pollution in marine sediments of the United Arab Emirates creeks along the Arabian Gulf shoreline. Bull. Environ. Contam. Toxicol. 60 417-424.

SKELTON PS (2001) A Complete Guide to the Freshwater Fishes of Southern Africa. Struik Publishers, Cape Town, South Africa.

SNODGRASS WJ (1980) Distribution and behavior of nickel in the aquatic environment. In: Nriagu JO (ed.) Nickel in the Environment. John Wiley and Sons, New York.

SORENSEN EM (1991) Metal Poisoning in Fish. CRC Press, Florida.

STEENKAMP VE, DU PREEZ HH and SCHOONBEE HJ (1994) Bioaccumulation of copper in the tissues of Potamonautes warreni (Calman) (Crustacea, Decapoda, Branchiura), from industrial, mine and sewage-polluted freshwater ecosystems. S. Afr. J. Zool. 29 (2) 152-161.

STRYDOM C, ROBINSON C, PRETORIUS E, WHITCUTT JM, MARX J and BORNMAN MS (2006) The effect of selected metals on the central metabolic pathways in biology: a review. Water $S A$ 32 (4) 543-554.

SU GS, MARTILLANO KJ, ALCANTARA TP, RAGRAGIO E, DE JESUS J, HALLARE A and RAMOS G (2009) Assessing heavy metals in the waters, fish and macroinvertebrates in Manila Bay, Philippines. J. Appl. Sci. Environ. Sanit. 4 (3) 187-195.

SULTANA R and RAO DP (1998) Bioaccumulation patterns of zinc, copper, lead and cadmium in grey mullet, Mugil cephalus (L.), from harbour waters of Visakhapatnam, India. Bull. Environ. Contam. Toxicol. 60 949-955.

SWALLOW KC, HUME DN and MOREL FMM (1980) Sorption of copper and lead by hydrous ferric oxide. Environ. Sci. Tech. 14 (11) 1326-1331.

UYSAL K, EMRE Y and KÖSE E (2008) The determination of heavy metal accumulation ratios in muscle, skin and gills of some migratory fish species by inductively coupled plasma-optical emission spectrometry (ICP-OES) in Beymelek Lagoon (Antalya/Turkey). Microchem. J. 90 67-70.

VAN DEN HEEVER DJ and FREY BJ (1994) Human health aspects of the metals zinc and copper in tissue of the African sharptooth catfish, Clarias gariepinus, kept in treated sewage effluent and in the Krugersdrift Dam. Water SA 20 (3) 205-212.

VAN DER MERWE CG, SCHOONBEE HJ and PRETORIUS J (1990) Observations on concentrations of the heavy metals zinc, manganese, nickel and iron in the water, in the sediments and in two aquatic macrophytes, Typha capensis (Rohrb.) N.E. Br. and Arundo donax L., of a stream affected by goldmine and industrial effluents. Water SA 16 (2) 119-124.

VAN LOON JC (1980) Analytical Atomic Absorption Spectroscopy; Selected Methods. Academic Press, New York.

VARIAN (1989) Analytical Methods in Flame Atomic Spectroscopy. Varian Techtron Pty Ltd, Mulgrave, Victoria, Australia.

VINODHINI R and NARAYANAN M (2008) Bioaccumulation of heavy metals in organs of fresh water fish Cyprinus carpio (Common carp). Int. J. Environ. Sci. Tech. 5 (2) 179-182.

VISNJIC-JEFTIC Z, JARIC I, JOVANOVIC L, SKORIC S, SMEDEREVAC-LALIC M, NIKCEVIC M and LENHARDT M (2010) Heavy metal and trace element accumulation in muscle, liver and gills of the Pontic shad (Alosa immaculate Bennet 1835) from the Danube River (Serbia). Microchem. J. 95 341-344.

WANG W (1987) Factors affecting metal toxicity to (and accumulation by) aquatic organisms - overview. Environ. Int. 13 437-457.

WARD SM and NEUMANN RM (1999) Seasonal variations in concentrations of mercury in axial muscle tissue of largemouth bass. N. Am. J. Fish. Man. 19 89-96.

WOELFL S, MAGES M and TORRES P (2008) Trace metal concentrations in single specimens of the intestinal broad flatworm (Diphyllobothrium latum), compared to their fish host (Oncorhynchus mykiss) measured by total reflection X-ray fluorescence spectrometry. Spectrochim. Acta Part B 63 1450-1454.

WOODWARD DF, BRUMBAUGH WG, DELONAY AJ, LITTLE EE and SMITH CE (1994) Effects on rainbow trout fry of a metalscontaminated diet of benthic invertebrates from the Clark Fork River, Montana. Trans. Am. Fish. Soc. 123 51-62.

YANG R, YAO T, XU B, JIANG G and XIN X (2007) Accumulation features of organochlorine pesticides and heavy metals in fish from high mountain lakes and Lhasa River in the Tibetan Plateau. Environ. Int. 33 151-156.

YILMAZ F, ÖZDEMIR N, DEMIRAK A and TUNA AL (2007) Heavy metal levels in two fish species Leuciscus cephalus and Lepomis gibbosus. Food Chem. 100 830-835.

YILMAZ AB, SANGÜN MK, YAĞLIOĞLU D and TURAN C (2010) Metals (major, essential to non-essential) composition of the different tissues of three demersal fish species from İskenderun Bay, Turkey. Food Chem. 123 410-415.

YOSHITOMI T, KOYAMA J, IIDA A, OKAMOTO N and IKEDA Y (1998) Cadmium-induced scale deformation in carp (Cyprinus carpio). Bull. Environ. Contam. Toxicol. 60 639-644.

ZOU E (1997) Effects of sublethal exposure to zinc chloride on the reproduction of the water flea, Moina irrasa (Cladocera). Bull. Environ. Contam. Toxicol. 58 437-441. 